\title{
PORTRAIT OF THE FOUR REGULAR SUPER-EXPONENTIALS TO BASE SQRT(2)
}

\author{
DMITRII KOUZNETSOV AND HENRYK TRAPPMANN
}

\begin{abstract}
We introduce the concept of regular super-functions at a fixed point. It is derived from the concept of regular iteration. A super-function $\mathrm{F}$ of $\mathrm{h}$ is a solution of $\mathrm{F}(\mathrm{z}+1)=\mathrm{h}(\mathrm{F}(\mathrm{z}))$. We provide a condition for $\mathrm{F}$ being entire, we also give two uniqueness criteria for regular super-functions.

In the particular case $\mathrm{h}(\mathrm{x})=\mathrm{b}^{\wedge} \mathrm{x}$ we call $\mathrm{F}$ super-exponential. $\mathrm{h}$ has two real fixed points for $b$ between 1 and $\mathrm{e}^{\wedge}(1 / \mathrm{e})$. Exemplary we choose the base $\mathrm{b}=\operatorname{sqrt}(2)$ and portray the four classes of real regular super-exponentials in the complex plane. There are two at fixed point 2 and two at fixed point 4 . Each class is given by the translations along the $\mathrm{x}$-axis of a suitable representative.

Both super-exponentials at fixed point 4-one strictly increasing and one strictly decreasing - are entire. Both super-exponentials at fixed point 2one strictly increasing and one strictly decreasing - are holomorphic on a right half-plane. All four super-exponentials are periodic along the imaginary axis. Only the strictly increasing super-exponential at 2 can satisfy $F(0)=1$ and can hence be called tetrational.

We develop numerical algorithms for the precise evaluation of these functions and their inverses in the complex plane. We graph the two corresponding different half-iterates of $h(z)=\operatorname{sqrt}(2)^{\wedge} z$. An apparent symmetry of the tetrational to base $\operatorname{sqrt}(2)$ disproved.
\end{abstract}

\section{SUPER-FUNCTIONS}

The functional equation

$$
F(z+1)=h(F(z))
$$

refers to the building-up of a super-function $F$ of some given base-function $h$. Multiplication $F(x)=b x$ is a super-function of addition $h(x)=b+x$. Exponentiation $F(x)=b^{x}$ is a super-function of multiplication $h(x)=b x$. Such an equation also occurs in the phenomenological description of a nonlinear optical fiber of fixed length [11, where $F(z)$ represents some parameter (for example, the logarithm of the power) at distance $z$ from the input tip, and $h$ is the transfer function. In that application $z$ is supposed to be real. In general, we assume that equation (1.1) holds for $z \in C \subseteq \mathbb{C}$, and $F$ is holomorphic on $C$.

Definition 1 (super-function, base-function, super-exponential, tetrational). Let $C$ be a domain such that for each $z \in C$ also $z+1 \in C$. A function $F$ defined on $C$ is called a super-function of $h$, if it satisfies equation (1.1) for all $z \in C$. We call $h$ the base-function of $F$. Applying the prefix $a \mapsto d$ (to "super-function") means that

Received by the editor June 1, 2009 and, in revised form, August 9, 2009.

2000 Mathematics Subject Classification. Primary 30A99; Secondary 33F99.

(C)2010 American Mathematical Society Reverts to public domain 28 years from publication 
additionally $F(a)=d$. Sometimes we also include the domain of holomorphism $C$ into the prefix, writing $(C, a \mapsto d)$, which additionally means that $F$ is holomorphic on $C$. Any super-function of $h(z)=b^{z}$ is called super-exponential to base $b$. Any $0 \mapsto 1$ super-exponential to base $b$ is called tetrational to base $b$.

The name super-exponential is due to Bromer 6], although other names (generalized exponential, iterated exponential, tetration, ultra-exponential, etc.) are also used for the iteration of an exponential function [10, 9, 3, 16.

In order to illustrate the definition of super-function, we suggest a few examples below.

Let $h(z)=b+z$ on $z \in \mathbb{C}$. Then, the function $F(z)=b \cdot z$ on $z \in \mathbb{C}$ is a $(\mathbb{C}, 0 \mapsto 0)$ super-function of $h$.

Now let $h(z)=b \cdot z$ on $z \in \mathbb{C}$. Then $F(z)=\exp _{b}(z)=b^{z}$ is a $(\mathbb{C}, 0 \mapsto 1)$ super-function of $h$.

Now we let the previous super-function again be the base-function $h=\exp _{b}$. For $C=\mathbb{C} \backslash\{x \in \mathbb{R}: x \leq-2\}$, the tetrational $F$ by [11] is a $(C, 0 \mapsto 1)$ super-function of exp- or super-exponential to base e.

For some base-functions it is possible to obtain elementary super-functions by using special functional relations, for example, $F(z)=\cos \left(\pi 2^{z}\right)$ is a $(\mathbb{C}, 0 \mapsto-1)$ super-function of the quadratic base-function $h(z)=2 z^{2}-1$. More trigonometric examples can be found in [18. Also, for real $j>0$, and base-function $h(z)=z^{j}$ the function $F(z)=\exp \left(j^{z}\right)$ is a super-function.

No representation through elementary or special functions is reported for nonconstant super-exponentials yet. So it would make sense to lift them into the status of new special functions after clarifying the uniqueness situation (similar to the complex extension of the factorial through the Gamma function).

Without additional conditions a super-function is not unique, generally. If $F$ is a $(C, a \mapsto d)$ super-function of $h$, then for any 1-periodic holomorphic function $\theta$, such that $\theta(a)=0$, the function $E(z)=F(z+\theta(z))$ will be a $(D, a \mapsto d)$ super-function of $h$ for $D=\{z: z+\theta(z) \in C\}$ :

$$
\begin{aligned}
E(a) & =F(a+\theta(a))=d, \\
E(z+1) & =F(z+\theta(z+1)+1)=h(F(z+\theta(z)))=h(E(z)) .
\end{aligned}
$$

However, the range $D$ of holomorphism of the function $E$ may be different from $C$. Here is a question how an appropriately large domain of holomorphism restricts the possible solutions. We give a uniqueness criterion in Proposition 7.

This paper is motivated by discussions on 17] about a generalization of the algorithm [11] for a holomorphic tetrational, which seems to work for various values $b>\exp (1 / \mathrm{e})$, in particular, for $b=2$, see [12, 19, but cannot be applied "as is" for the case $1<b \leq \exp (1 / \mathrm{e})$. In order to elaborate a robust algorithm for the evaluation of the tetrational in this case, we consider one specific value $b=\sqrt{2}$ from this interval. We consider not only the tetrational, but also some super-exponentials to this base which can be easily generalized to bases $1<b<\exp (1 / \mathrm{e})$.

In order to be explicit, we look for holomorphic, real solutions $F$ of the equation

$$
F(z+1)=\exp _{b}(F(z))
$$

for the specific value $b=\sqrt{2}$. (We call any $C \mapsto \mathbb{C}$ function $f$ real, if $f\left(z^{*}\right)=$ $f(z)^{*} \forall z \in C$.) 


\section{SUPER-FUnCtions By REgUlar iteration}

In this section we assume, that the function $h$ has some fixed point $\lambda$, i.e., $h(\lambda)=\lambda$. For example, $\lambda=2$ and $\lambda=4$ are the only real fixed points of $h=\exp _{\sqrt{2}}$ and $\lambda=\mathrm{e}$ is the only real fixed point of $h=\exp _{\exp (1 / \mathrm{e})}$. Assume that the function $h$ is holomorphic at least in some vicinity of the fixed point $\lambda$. Then, a superfunction $F$ of $h$ can be constructed (under certain additional conditions), such that $F$ approaches $\lambda$ asymptotically, at least in some directions in the complex plane.

The holomorphic function $h$ can be expanded in a series that converges in some domain around the fixed point

$$
f(z)=h(\lambda+z)-\lambda=\sum_{n=1}^{\infty} f_{n} z^{n} .
$$

In this paper, for a function $f$ we use notation $f_{n}$ for the $n$th Taylor coefficient at $\lambda$. The formal solution $F$ with exponential behavior can be constructed in the following way. Let

$$
F(z)=\lambda+G(\mathcal{E}(z))
$$

for some function $G$ expandable into a series

$$
G(z)=z+\sum_{n=2}^{\infty} G_{n} z^{n}
$$

and

$$
\mathcal{E}(z)=\exp ((z+t) \kappa)=s \exp (\kappa z)
$$

for some constant parameters $t$ and $\kappa$, where $s=\mathrm{e}^{\kappa t}$. Substitution $z \mapsto z+1$ in equation (2.2) gives

$$
F(z+1)=\lambda+G(K \mathcal{E}(z))
$$

where $K=\exp (\kappa)$. Then, equation (1.1) can be written as

$$
\begin{aligned}
\lambda+G(K \mathcal{E}(z)) & =h(\lambda+G(\mathcal{E}(z))), \\
\lambda+G(K \omega) & =h(\lambda+G(\omega)) .
\end{aligned}
$$

The Taylor expansion of both sides of (2.7) at $\omega=0$ leads to the equalities for the coefficients

$$
\begin{aligned}
K & =f_{1}, \\
G_{2} K^{2} & =f_{1} G_{2}+f_{2}, \\
G_{3} K^{3} & =f_{1} G_{3}+2 f_{2} G_{2}+f_{3}, \\
G_{4} K^{4} & =f_{1} G_{4}+3 f_{3} G_{2}+2 f_{2} G_{3}+f_{2} G_{2}^{2}+f_{4} .
\end{aligned}
$$

It seems as if $G_{n}$ can be determined without solving polynomial equations. This is indeed the case and it turns out that this approach is equivalent to the well-known approach of regular iteration via the Schröder equation [15, 14, 7].

A few notes about notation. For any (formal) powerseries $f$ we denote the coefficients by $f_{n} ; f(z)=\sum_{n=0}^{\infty} f_{n} z^{n}$ (although the series has no need to converge.) 
We write $f^{m}$ for the $m$ th power of the function $f$, and $f^{[m]}$ for the $m$ th iterate of $f$, i.e.,

$$
f^{[m]}(z)=\underbrace{f(f(\ldots f(z) \ldots))}_{m \text { evaluations of } f .}
$$

For example, below $f_{n}^{m}$ means the $n$th Taylor coefficient of the function that is the $m$ th power of function $f$. It should not be confused with $f_{n}^{m}$, which is the $m$ th power of the $n$th Taylor coefficient, or with $f_{n}^{[m]}$, which is the $n$th coefficient of the expansion of the $m$ th iterate of function $f$ at 0 . There are few exceptions to this rule. First, we keep the usual notation $F^{-1}$ for the inverse function of $F$, although it is actually minus the first iteration of function $F$; and of course we use subscripts as usual on non-functions, like $\kappa_{2}$ or $T_{4}$. You can find a table of notation in the appendix; we tried to distribute the semantic load uniformly among the letters of the Latin alphabet, but we use a few Greek letters too.

Definition 2 (Schröder equation, Schröder function). For a function $h$ with fixed point $\lambda$ and $K=h^{\prime}(\lambda)$, we call any function $\chi$ that satisfies the Schröder equation

$$
\chi(h(z))=K \chi(z)
$$

a Schröder function of $h$ at $\lambda$.

For ease of derivation we first assume that the fixed point is 0 . If we want the Schröder function $\chi$ to be analytic, then it is already determined by $\chi^{\prime}(0)=1$. We show this in the next proposition.

Proposition 1 (Schröder powerseries). Let $f$ be a formal powerseries with $f_{0}=0$ (fixed point 0 ) and $f_{1}^{n} \neq f_{1}$ for all integer $n>1$ (particularly if $\left|f_{1}\right| \neq 0,1$ ). Then there is a unique formal powerseries solution $\chi$ with $\chi_{1}=1$ of the Schröder equation

$$
\chi(f(z))=f_{1} \chi(z) .
$$

We call this solution the Schröder powerseries of $f$. The Schröder powerseries $\chi$ and its inverse $\eta=\chi^{-1}$ are given by $\chi_{0}=\eta_{0}=0, \chi_{1}=\eta_{1}=1$, and, for $n>1$, by the recursive formulas

$$
\chi_{n}=\frac{1}{f_{1}-f_{1}{ }^{n}} \sum_{m=1}^{n-1} \chi_{m} f^{m}{ }_{n}, \quad \eta_{n}=\frac{1}{f_{1}{ }^{n}-f_{1}} \sum_{m=2}^{n} f_{m} \eta^{m}{ }_{n}
$$

where the $m$ th power of an arbitrary formal powerseries $g$ is defined by

$$
g_{n}^{m}=\sum_{n_{1}+\cdots+n_{m}=n} g_{n_{1}} \ldots g_{n_{m}} .
$$

Proof. The recursive solutions are a direct consequence of the composition formula of two formal powerseries $f$ and $g$ with $g_{0}=0$ :

$$
(f \circ g)_{n}=\sum_{m=0}^{n} f_{m} g_{n}^{m}
$$

applied to our Schröder equation (2.14) or its inverse $\eta\left(f_{1} z\right)=f(\eta(z))$ :

$$
f_{1} \chi_{n}=\sum_{m=0}^{n} \chi_{m} f_{n}^{m}, \quad f_{1}^{n} \eta_{n}=\sum_{m=1}^{n} f_{m} \eta_{n}^{m} .
$$


First we can observe at the left side that $f_{1} \chi_{0}=\chi_{0}$, which implies $\chi_{0}=0$ for $f_{1} \neq 0,1$. In consequence $\eta_{0}=0$. For the indices above 1 we rearrange the equations to get the $\chi_{n}$ or $\eta_{n}$ that occur in the sum of the right side to the left side and where we take into account that $f_{n}^{n}=f_{1}^{n}$ and $\eta_{n}^{1}=\eta_{n}$.

Proposition 2. Every formal powerseries $\mathcal{X}$ solution of (2.14) can be expressed as $\mathcal{X}(z)=s \chi(z)$ for some $s \in \mathbb{C}$.

Proof. Setting $\mathcal{X}_{1}=s$ in the above derivation, one inductively gets $\mathcal{X}_{n}=s \chi_{n}$.

Now we apply this to our original problem (2.7). We see that $G$ is just the inverse Schröder function of $h(z+\lambda)-\lambda$. Let us write down the first few coefficients of $G$.

$$
\begin{gathered}
G_{0}=0, \quad G_{1}=1, \\
G_{2}=\frac{1}{f_{1}^{2}-f_{1}} f_{2} g_{2}^{2}=\frac{f_{2}}{f_{1}^{2}-f_{1}}, \\
G_{3}=\frac{f_{2}\left(\sum_{n_{1}+n_{2}=3} G_{n_{1}} G_{n_{2}}\right)+f_{3} G_{3}^{3}}{f_{1}^{3}-f_{1}}=\frac{f_{2}\left(2 G_{1} G_{2}\right)+f_{3}}{f_{1}^{3}-f_{1}} \\
=\frac{2 f_{2} \frac{f_{2}}{f_{1}^{2}-f_{1}}+f_{3}}{f_{1}^{3}-f_{1}}=\frac{2 f_{2} f_{2}+f_{3} f_{1}^{2}-f_{3} f_{1}}{\left(f_{1}^{3}-f_{1}\right)\left(f_{1}^{2}-f_{1}\right)}=\frac{2 f_{2}^{2}+f_{3} f_{1}^{2}-f_{3} f_{1}}{\left(f_{1}-1\right)\left(f_{1}^{2}-1\right) f_{1}^{2}} .
\end{gathered}
$$

Proposition 3. If the powerseries $f$ with $f_{0}=0$ and $\left|f_{1}\right| \neq 0,1$ has non-zero convergence radius, then the Schröder powerseries $\chi$ of $f$ has non-zero convergence radius also.

Proof. For a proof you can see [14], Theorem 4.6.1.

Proposition 4. Let $f$ be holomorphic in a vicinity of $0, f(0)=0$, set $K=f^{\prime}(0)$, let $|K| \neq 0,1$. If $f$ is entire and $|K|>1$ or if $f^{-1}$ is entire and $|K|<1$, then the inverse Schröder function of $f$ (i.e. the function with the inverse Schröder powerseries development at 0 ) is entire.

Proof. We continue the inverse Schröder function to the whole complex plane. By Proposition 3 there is a disk $D_{\delta}$ with radius $\delta>0$ at 0 where the inverse Schröder function $\eta$ of $f$ is analytic. We then define $\hat{\eta}$ on the disk with radius $|K|^{ \pm 1} \delta$ for all $z \in D_{\delta}$ via

$$
\begin{aligned}
& \hat{\eta}(K z):=f(\eta(z)) \quad \text { for } \quad|K|>1 \text {, } \\
& \hat{\eta}(z):=f^{-1}(\eta(K z)) \quad \text { for } \quad|K|<1 \text {. }
\end{aligned}
$$

$\hat{\eta}$ coincides by the Schröder equation with $\eta$ on $D_{\delta}$ and is hence a continuation to $D_{|K|^{ \pm 1} \delta} \supset D_{\delta}$. We repeatedly apply this process to continue $\eta$ to the disk with radius $|K|^{ \pm n} \delta$ and so continue $\eta$ to the whole complex plane.

Proposition 5. Let $f$ be analytic at the fixed point 0 , set $K=f^{\prime}(0)$ and assume $|K| \neq 0,1$. Then, the Schröder powerseries $\chi$ of $f$ can be analytically continued to the basin of attraction $\Omega(f)=\left\{z: \lim _{n \rightarrow \infty} f^{[n]}(z)=0\right\}$ of $f$ at 0 (for $|K|<1$ ) or to $\Omega\left(f^{-1}\right)$ (for $|K|>1$ ), and the convergence radius $R$ of $\chi$ is bounded by

$$
R \geq \sup \left\{r>0: B_{r}(0) \subseteq \Omega\left(f^{[ \pm 1]}\right)\right\} .
$$


Proof. By the equation $\chi\left(f^{[n]}(z)\right)=K^{n} \chi(z)$ we get a rule to continue $\chi$ to $\Omega\left(f^{ \pm 1}\right)$ :

$$
\chi(z)=\frac{\chi\left(f^{[ \pm n]}(z)\right)}{K^{ \pm n}} .
$$

We saw that we can express a super-function $F$ of $f$ through the inverse Schröder function: $F(z)=\eta\left(s \mathrm{e}^{k z}\right)$. As mentioned in the introduction, there is a wide range of other $a \mapsto d$ super-functions: If $F$ is a holomorphic $a \mapsto d$ super-function of $f$, then for every holomorphic 1-periodic function $\theta$ with $\theta(c)=0$ the function $E(z)=F(z+\theta(z))$ is another holomorphic $a \mapsto d$ super-function of $f$. Therefore, a condition, that singles out the solutions $\eta\left(s \mathrm{e}^{\kappa z}\right)$, would be quite welcome. Such a condition is given in the next two propositions.

Proposition 6. Let $\kappa$ be a complex number with $\Re(\kappa) \neq 0$. Then for each $s \in \mathbb{C}$ the function $F(z)=s \mathrm{e}^{\kappa z}$ is the only $0 \mapsto s$ solution of $F(z+1)=\mathrm{e}^{\kappa} F(z)$, that is, holomorphic and bounded on some strip $S_{x, \varepsilon}=\{z / \kappa+x-\xi: 0 \leq \xi<1+\varepsilon, \Re(z)=0\}$ (which is the strip between the straight lines orthogonal to $\bar{\kappa}$ going through the points $x$ and $x-1-\varepsilon)$.

Proof. First $F(z)=s \mathrm{e}^{\kappa z}$ is indeed a $0 \mapsto s$ solution and it is bounded on $w \in S_{x, \varepsilon}$ for any fixed $x$ and $\varepsilon>0$ because $\left|s e^{\kappa w}\right|=|s| \mathrm{e}^{\Re(z+\kappa(x-\xi))}=|s| \mathrm{e}^{\Re(\kappa)(x-\xi)} \leq$ $|s| \mathrm{e}^{|\Re(\kappa)|(1+\varepsilon+|x|)}$.

$F_{1}(z):=\mathrm{e}^{\kappa z}$ is also bounded from below by $\left|F_{1}(z)\right| \geq \mathrm{e}^{-|\Re(\kappa)|(|x|+1+\varepsilon)}$. Let $\tilde{F}$ be another solution, consider $\theta(z)=\tilde{F}(z) / F_{1}(z)$ which must be bounded on $S_{x, \varepsilon}$. Then $\theta(z+1)=\theta(z), \theta$ is a periodic function on $S_{x, \varepsilon}$. The translations of the strip $S_{x, \varepsilon}$ by integers $k$ overlappingly cover $\mathbb{C}, \bigcup_{k \in \mathbb{Z}}\left(S_{x, \varepsilon}+k\right)=\mathbb{C}$. (Note that this is not the case for $\Re(\kappa)=0$.) Then, function $\theta$ can be holomorphically continued to $\mathbb{C}$ by $\theta(z+k)=\theta(z)$. Now $\theta$ is entire and bounded and hence constant: $\tilde{F}(z)=s F_{1}(z)$, where $s$ must be $\tilde{F}(0)$.

The following proposition characterizes the solutions $\eta\left(s \mathrm{e}^{\kappa z}\right)$ as those which satisfy a certain asymptotic property in the direction $\kappa$ of the complex plane.

Proposition 7. Let $f$ be analytic at $0, f(0)=0, K:=f^{\prime}(0)$, and $|K| \neq 0,1$, let $\kappa$ be some number with $\mathrm{e}^{\kappa}=K$, and let $H_{\kappa, x}:=\{z / \kappa: \Re(z) \leq x\}$ be the halfplane bounded by the straight line going through and being orthogonal to $x / \kappa$. Then the set of any $H_{\kappa, x}$ holomorphic and bounded super-functions $F$ of $f$ which satisfy $\lim _{x \rightarrow \infty} \sup \left|F\left(H_{\kappa,-x}\right)\right|=0$ consists exactly of the functions

$$
F(z)=\eta\left(s \mathrm{e}^{\kappa z}\right), \quad s \in \mathbb{C},
$$

where $\eta$ is the inverse Schröder powerseries of $f$.

Proof. First, each $F(z)=\eta\left(s \mathrm{e}^{\kappa z}\right)$ is clearly a holomorphic super-function of $f$. It is bounded, because $s \mathrm{e}^{\kappa z}$ maps $H_{\kappa, x}$ to the closed disk $|z| \leq|s| \mathrm{e}^{x}$, where $\eta$ is holomorphic (continuous) and hence bounded.

Now let $F$ be arbitrary on $H_{\kappa, x}$ holomorphic and a bounded super-function of $f$ which satisfies $\lim _{x \rightarrow \infty} \sup \left|F\left(H_{\kappa,-x}\right)\right|=0$; consider the function $g(z)=\eta^{-1}(F(z))$. Choosing $x$ small enough, by $\lim _{x \rightarrow \infty} \sup \left|F\left(H_{\kappa,-x}\right)\right|=0$ we can ascertain that $F(z)$ has values only within the convergence disk of $\eta^{-1}$ for $z \in H_{\kappa, x}$. So $g$ is holomorphic, and bounded on $H_{\kappa, x}$, and satisfies

$$
g(z+1)=\eta^{-1}(f(F(z)))=K \eta^{-1}(F(z))=K g(z)
$$


for all $z$ such that $z, z+1 \in H_{\kappa, x}$. As $|K| \neq 1$, we know that $\Re(\kappa) \neq 0$; and from Proposition [6 it follows, that $g(z)=s \mathrm{e}^{\kappa z}$ and hence $F(z)=\eta\left(s \mathrm{e}^{\kappa z}\right)$.

Now we switch from the fixed point 0 to an arbitrary fixed point $\lambda$. If $\lambda$ is a fixed point of $h$, then 0 is a fixed point of $z \mapsto h(z+\lambda)-\lambda$. It is clear, that, if $\chi$ is a Schröder function (meaning that is satisfies the Schröder equation) of $h$, then $z \mapsto \chi(z+t)$ is a Schröder function of $z \mapsto h(z+t)-t$ for any $t$;

$$
\chi((h(z+t)-t)+t)=\chi(h(z+t))=K \chi(z+t)
$$

and vice versa. If $\eta$ is an inverse Schröder function of $h$, then $z \mapsto \eta(z)-t$ is an inverse Schröder function of $z \mapsto h(z+t)-t$ and

$$
\eta(K z)-t=h(\eta(z))-t=h((\eta(z)-t)+t)-t .
$$

Definition 3 (regular and principal super-function). Let $h$ be analytic at its fixed point $\lambda$ and $\mathrm{e}^{\kappa}=h^{\prime}(\lambda), \Re(\kappa) \neq 0$. We call the functions $z \mapsto \eta\left(s \mathrm{e}^{\kappa z}\right)+\lambda, s \in \mathbb{C}$, the $\kappa$-regular super-functions of $h$ at $\lambda$, where $\eta$ is the inverse Schröder powerseries of $z \mapsto h(z+\lambda)-\lambda$. We call the function $z \mapsto \eta\left(\mathrm{e}^{\kappa z}\right)+\lambda$ the $\kappa$-principal super-function of $h$ at $\lambda$. Omitting $\kappa$ above means $\kappa=\log \left(h^{\prime}(\lambda)\right)$ for the standard branch of the logarithm.

Corollary 8. Every $\kappa$-regular super-function $F$ is periodic with period $T=2 \pi \mathrm{i} / \kappa$ and $F(z)$ is a translation of the $\kappa$-principal super-function $\mathcal{F}(z)$ along the $z$-plane, i.e. $F(z)=\mathcal{F}(z+t)$ for some $t \in \mathbb{C}$. We can always choose $t=\log (s) / \kappa$, where $s$ is taken from the previous definition.

Corollary 9. Let $h$ be analytic at $\lambda, h(\lambda)=\lambda, K:=h^{\prime}(\lambda)$, such that $|K| \neq 0,1$, let $\kappa$ be some number with $e^{\kappa}=K$, and let $H_{\kappa, x}$ be as in Proposition 7, Then the set on some $H_{\kappa, x}$ holomorphic and bounded super-functions $F$ of $h$ which satisfy $\lim _{x \rightarrow \infty} \sup \left|F\left(H_{\kappa,-x}\right)-\lambda\right|=0$ is the set of the $\kappa$-regular super-functions of $h$ at $\lambda$.

Let us now consider strictly increasing real-analytic super-functions. We know that the inverse Schröder function of a real-analytic function $h$ is strictly increasing in a vicinity of the fixed point because $\eta^{\prime}(0)=1$, and we want to define the superfunction as $F(x)=\eta\left(s \mathrm{e}^{\kappa x}\right)+\lambda$.

There is a natural distinction between positive and negative $s$. (We exclude the trivial case $s=0$, which gives the constant function $F(x)=\lambda$ ). Different $s$ with the same sign just translate the graph of $F$ along the $x$-axis; $\pm|s| \mathrm{e}^{\kappa x}= \pm \mathrm{e}^{\kappa(x+\ln |s| / \kappa)}$. The change of sign of $s$ is more dramatic; it corresponds to a translation along the imaginary axis and inverses the monotony. We use this for a classification of the regular super-functions of real base-functions:

Definition 4 (super-function above and below). Let $h$ be real-analytic at the fixed point $\lambda=h(\lambda)$ such that $0<h^{\prime}(\lambda) \neq 1$. Let $\kappa=\ln \left(h^{\prime}(\lambda)\right)$. Let $\eta$ be the inverse Schröder powerseries of $x \mapsto h(x+\lambda)-\lambda$. Then, we call the function

$$
F(z)=\eta\left(s \mathrm{e}^{\kappa z}\right)+\lambda
$$

a super-function of $h$ below $\lambda$, for each $s<0$; a super-function equal to $\lambda$ (i.e. the constant function $F(z)=\lambda$ ), for $s=0$; and a super-function above $\lambda$, for each $s>0$. 
Let $I$ be the maximal interval around $\lambda$ such that $\eta$ is analytically continuable to $I$ and strictly increasing there. For any $d \in \eta(I)+\lambda$ and

$$
s=\eta^{-1}(d-\lambda)
$$

we call the function

$$
F(z)=\eta\left(s \mathrm{e}^{\kappa z}\right)+\lambda
$$

the regular $0 \mapsto d$ super-function $F$ of $h$ at $\lambda$.

The reader may verify that these functions are indeed all possible real-analytic regular super-functions.

Let $F(x)=\eta\left(s \mathrm{e}^{\kappa x}\right)+\lambda$, i.e. as in the previous definition. Let the word "finally" mean: "there exist an $x_{0}$ such that for all $x>x_{0}$ " in the case $\kappa<0$, and "there exist an $x_{0}$ such that for all $x<x_{0}$ " in the case $\kappa>0$. Then we can apply our previous propositions and state:

(1) For all $\varepsilon>0$ finally $|F(x)-\lambda|<\varepsilon$.

(2) Finally $F(x)>\lambda$ if $s>0$.

(3) Finally $F(x)<\lambda$ if $s<0$.

(4) Finally $F$ is strictly increasing if $\kappa s>0$.

(5) Finally $F$ is strictly decreasing if $\kappa s<0$.

(6) $F$ is holomorphic on a right half-plane if $\kappa<0$.

(7) $F$ is entire if $\kappa>0$.

(8) $F$ is periodic with the imaginary period $T=2 \pi \mathrm{i} / \kappa$.

Actually the conditions (1), (6) and (8) already determine $F$ to be a regular superfunction, as we show in the next proposition for the case $\kappa<0$. This is a supplemental uniqueness criterion to the one given in Proposition 7 and Corollary 9 .

Proposition 10. Let $h$ be a function, real-analytic in a vicinity of its fixed point $\lambda$, with $0<h^{\prime}(\lambda)<1$; let $\kappa=\ln \left(h^{\prime}(\lambda)\right)$. Each real-analytic super-function $F$ holomorphic on a right half-plane $H_{x}=\{z \in \mathbb{C}: \Re(z)>x\}$ and $2 \pi \mathrm{i} / \kappa$ periodic that satisfies $\lim _{x \rightarrow \infty} F(x)=\lambda$ is a regular super-function.

Proof. Let $G(z)=\eta\left(\mathrm{e}^{\kappa z}\right)+\lambda$ be the principal super-function of $h$. It is finally either strictly increasing or strictly decreasing, hence finally injective. As $\lim _{x \rightarrow \infty} F(x)=$ $\lambda$ there is an $x_{0}$ such that

$$
W(x)=G^{-1}(F(x))
$$

is well defined for $x>x_{0}$. It satisfies

$$
W(x+1)=G^{-1}(h(F(x)))=W(x)+1
$$

and we can express $F$ with $G$ and $W$ for $x>x_{0}$ :

$$
F(x)=G(W(x))=\eta\left(\mathrm{e}^{\kappa W(x)}\right)+\lambda .
$$

Now we have the precondition that $F$ is $2 \pi \mathrm{i} / \kappa$ periodic. We know also that $\eta(x)$ is injective in a vicinity $\varepsilon$ of 0 . Hence we possibly increase $x_{0}$ somewhat so that $\mathrm{e}^{\kappa x}<\varepsilon$ for $x>x_{0}$. We then apply the injectivity of $\eta$ on the periodic equality:

$$
\begin{aligned}
\eta(\exp (\kappa W(x+2 \pi \mathrm{i} / \kappa)))+\lambda & =\eta(\exp (\kappa W(x)))+\lambda, \\
\exp (\kappa W(x+2 \pi \mathrm{i} / \kappa)) & =\exp (\kappa W(x)), \\
\kappa W(x+2 \pi \mathrm{i} / \kappa) & =\kappa W(x)+2 \pi \mathrm{i} N,
\end{aligned}
$$


for some integer $N$. So $W$ has the property

$$
W\left(x+\frac{2 \pi \mathrm{i}}{\kappa}\right)=W(x)+\frac{2 \pi \mathrm{i}}{\kappa} N,
$$

By (2.26) $\theta(x)=W(x)-x$ is 1-periodic. It has a Fourier development, which means that there is a Laurent series $\rho$ at 0 such that

$$
\begin{gathered}
W(z)-z=\theta(z)=\rho\left(\mathrm{e}^{2 \pi \mathrm{i} z}\right), \\
W(z)=\rho\left(\mathrm{e}^{2 \pi \mathrm{i} z}\right)+z .
\end{gathered}
$$

Now we plug this into equation (2.28) and derive

$$
\begin{aligned}
\rho\left(\mathrm{e}^{2 \pi \mathrm{i}(z+2 \pi \mathrm{i} / \kappa)}\right)+z+\frac{2 \pi \mathrm{i}}{\kappa} & =\rho\left(\mathrm{e}^{2 \pi \mathrm{i} z}\right)+z+\frac{2 \pi \mathrm{i}}{\kappa} N, \\
\rho\left(\mathrm{e}^{2 \pi \mathrm{i} z} e^{-4 \pi^{2} / \kappa}\right) & =\rho\left(\mathrm{e}^{2 \pi \mathrm{i} z}\right)+\frac{2 \pi \mathrm{i}}{\kappa}(N-1), \\
\rho\left(z \mathrm{e}^{-4 \pi^{2} / \kappa}\right) & =\rho(z)+\frac{2 \pi \mathrm{i}}{\kappa}(N-1) .
\end{aligned}
$$

As the Laurent series development is unique, we obtain the equations

$$
\rho_{n} \mathrm{e}^{-4 n \pi^{2} / \kappa}=\rho_{n}
$$

for $n \neq 0$ which implies $\rho_{n}=0$ and

$$
\rho_{0}=\rho_{0}+\frac{2 \pi \mathrm{i}}{\kappa}(N-1)
$$

which implies $N=1$. Hence $\rho(x)=\rho_{0}$ is constant, then $W(x)=x+\rho_{0}$, then $F(x)=G\left(x+\rho_{0}\right)$, which actually means that $F$ is a regular super-function of $h$; see Definition 3 ,

Next we apply the previous propositions and statements to $h=\exp _{\sqrt{2}}$ with the two fixed points $\lambda=2,4$.

\section{Super-eXPonential BELOW FIXed POINT 2}

For base $b>\exp (1 / \mathrm{e})$ a super-exponential can be evaluated through the Cauchy integral, that gives the integral equation for values of the function along the imaginary axis [1]. For $b=\sqrt{2}$, this algorithm cannot be applied as is, because both fixed points $\lambda=2$ and $\lambda=4$ are real; but regular iteration can be applied for precise evaluation.

Throughout this section, the base is $b=\sqrt{2}, h=\exp _{b}$; we consider the fixed point $\lambda=2$, so,

$$
\begin{aligned}
K_{2} & =h^{\prime}(\lambda)=\exp _{\sqrt{2}}(2)=2 \ln (\sqrt{2})=\ln (2) \approx 0.69314718055995, \\
\kappa_{2} & =\ln \left(K_{2}\right)=\ln (\ln (2)) \approx-0.36651292058166 .
\end{aligned}
$$

With formula (2.15) we compute the coefficients of the inverse Schröder function

$$
v(z)=z+\sum_{n=2}^{\infty} v_{n} z^{n}
$$


TABLE 1. Evaluation of coefficients $v$ and $V$ in (3.3), (3.18)

$\begin{array}{rrc}n & v_{n} & V_{n} \\ 1 & 1.0000000000000000 & 1.0000000000000000 \\ 2 & -0.5647228383177324 & 0.5647228383177324 \\ 3 & 0.3381775868511833 & 0.2996461813840881 \\ 4 & -0.2103313021386278 & 0.1559323904892543 \\ 5 & 0.1344548790521098 & 0.0803518797481544 \\ 6 & -0.0877843886012191 & 0.0411584960662439 \\ 7 & 0.0582880930830947 & 0.0209985209544120 \\ 8 & -0.0392407117837278 & 0.0106825803202636 \\ 9 & 0.0267232860342981 & 0.0054228810223159 \\ 10 & -0.0183765205976376 & 0.0027482526618683 \\ 11 & 0.0127420898467766 & 0.0013909151872678 \\ 12 & -0.0088986329515697 & 0.0007031815862125 \\ 13 & 0.0062531995639749 & 0.0003551700677648 \\ 14 & -0.0044181328624397 & 0.0001792537427482 \\ 15 & 0.0031365295362696 & 0.0000904088765718 \\ 16 & -0.0022361213774487 & 0.0000455725430285 \\ 17 & 0.0016001999145218 & 0.0000229602263218 \\ 18 & -0.0011489818761273 & 0.0000115627707503 \\ 19 & 0.0008274921384317 & 0.0000058201696570 \\ 20 & -0.0005975832172069 & 0.0000029289688393\end{array}$

of

$$
\begin{aligned}
h[2](z) & :=\exp _{\sqrt{2}}(z+2)-2=2\left(\exp \left(\frac{\ln (2)}{2} z\right)-1\right), \\
h[2](z) & =\sum_{n=1}^{\infty} \frac{\ln (2)^{n}}{2^{n-1} n !} z^{n}, \\
h[2]_{1} & =\ln (2) \\
h[2]_{2} & =\frac{\ln (2)^{2}}{4}, \\
h[2]_{3} & =\frac{\ln (2)^{3}}{24},
\end{aligned}
$$

and so on. As an example we compute $v_{2}$ and $v_{3}$ via (2.18) and (2.19):

$$
\begin{aligned}
& v_{2}=\frac{\ln (2) / 4}{\ln (2)-1} \quad \approx-0.56472283831773236365 \text {, } \\
& v_{3}=\frac{\ln (2)^{2}(2+\ln (2)) / 24}{(\ln (2)-1)\left(\ln (2)^{2}-1\right)} \approx 0.33817758685118329988 .
\end{aligned}
$$

More values for the coefficients $v$ are shown in Table 1 .

The regular $0 \mapsto 1$ super-exponential is then, by Definition 4

$$
\begin{aligned}
F_{2,1}(z) & =v\left(s_{2,1} \mathrm{e}^{\kappa_{2} z}\right)+\lambda=v\left(s_{2,1} \ln (2)^{z}\right)+2, \\
s_{2,1} & =V(1-\lambda)=V(-1) \approx-0.63209866105083,
\end{aligned}
$$

where $V$ is the inverse powerseries of $v$ (i.e. the Schröder powerseries of $h[2]$ ). Because $\kappa_{2}$ is negative the function $F_{2,1}(z)$ is holomorphic on some right half-plane (see the statement list at the end of Section 2). 

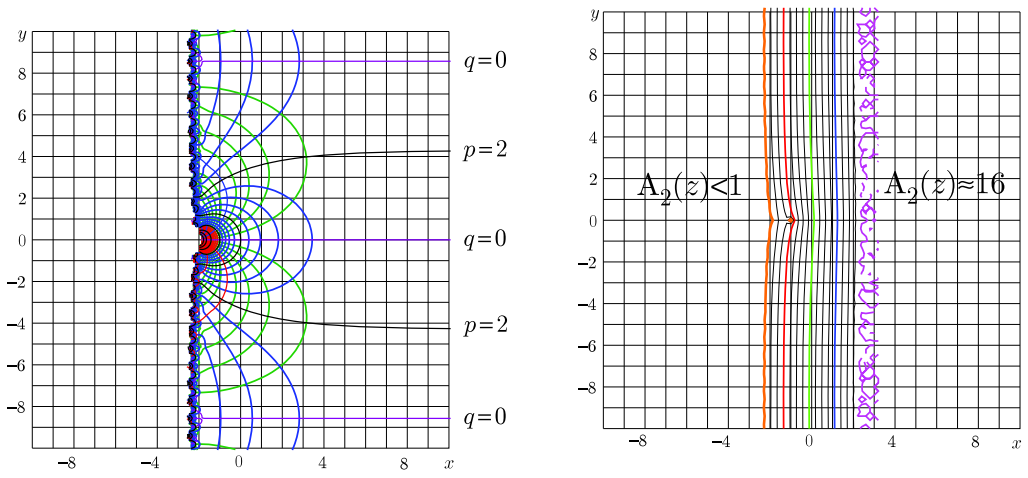

Figure 1. Function $f=\tilde{F}_{2,1,20}(z)$ by (3.11) in the $z=x+$ i $y$ plane: levels $p=\Re(f)=$ const and levels $q=\Im(f)=$ const are shown with thick lines for integer values of $p$ and $q$; left. Agreement $f=A_{2}(z)$ by (3.12) in the $z=x+\mathrm{i} y$ plane; levels $f=1,4,8,12,16$ are shown with thick lines; right.

The truncation of the series (3.3), keeping the last term at index $N$ gives a function, let us call it $\tilde{F}_{2,1, N}$, which approximates $F_{2,1}(z)$ at large positive values of $\Re(z)$ :

$$
\tilde{F}_{2,1, N}(z)=2+\sum_{n=1}^{N} v_{n} s_{2,1}^{n} \ln (2)^{z n} .
$$

For $N=20$, this function is shown in the left-hand side of Figure 1 with lines of constant real part and those of constant imaginary part. In the figure, the level $p=1$ visually goes through the origin of coordinates; in reality $\tilde{F}_{2,1,20}(0)$ slightly deviates from unity.

The precision of the approximation can be characterized with the agreement

$$
A_{2}(z)=-\lg \left|\frac{\tilde{F}_{2,1, N}(z)-\log _{b}\left(\tilde{F}_{2,1, N}(z+1)\right)}{\tilde{F}_{2,1, N}(z)+\log _{b}\left(\tilde{F}_{2,1, N}(z+1)\right)}\right|,
$$

Roughly, this agreement indicates, how many significant figures we may expect to get with this approximation. On the left-hand side, the agreement is smaller than unity; even the first decimal digit of the approximation is doubtful. On the righthand side, the agreement is of order of 16 . We expect, at $\Re(z)>3$ the precision of approximation $\tilde{F}_{2,1,20}(z)$ is limited mainly by the rounding errors. Even in the vicinity of the origin of coordinates, the approximation $\tilde{F}_{2,1,20}$ gives several correct decimal digits.

To evaluate $F_{2,1}(z)$ for various values $z$, the integer iterations can be used:

$$
F(z-k)=\log _{b}^{[k]}(F(z)) .
$$

For the complex $\langle$ double $\rangle$ implementation of $F_{2,1}$, we suggest the approximation

$$
\tilde{F}_{2,1}(z)=\left\{\begin{array}{cc}
\tilde{F}_{2,1,20}(z), & \Re(z)>3, \\
\log _{b}\left(\tilde{F}_{2,1}(z+1)\right), & \Re(z) \leq 3 .
\end{array}\right.
$$


This approximation is estimated to return at least 14 significant decimal digits; it is used to plot function $F_{2,1}$ in Figure2 2 in the same notations, as in Figure1. In the right-hand side of the plot, the function coincides with the primary approximation $\tilde{F}_{2,1,20}$. The approximation also reproduces the periodicity of $F_{2,1}$ :

$$
F_{2,1}\left(z+T_{2} n\right)=F_{2,1}(z), \quad T_{2}=\frac{2 \pi \mathrm{i}}{\kappa_{2}} \approx-17.1431481793548471 \mathrm{i}, n \in \mathbb{Z} .
$$

The function $F_{2,1}(z)$ has a branchpoint with logarithmic singularity at $z=-2$; we place the cut along the line $z<-2$. Due to the periodicity, there is a set of singularities and cut lines translated with period $T_{2}$ by (3.15). The other pictures in the Figure 2 represent functions considered in the following sections.

In Figure 3 we plot the function $F_{2,1}$ versus the real argument; see the thick curve at the bottom. The other curves correspond to the super-exponentials which we define and analyze in the following sections. We indicate the fixed point $\lambda$ as the first subscript of function $F$, and its value at zero as the second subscript. We choose this value as the natural number, closest to the fixed point. In such a way, we deal with the following super-exponentials:

$F_{2,1}$, developed at $\lambda=2$, with range of values $(-\infty, 2)$ along the real axis, $F_{2,3}$, developed at $\lambda=2$, with range of values $(2,4)$ along the real axis, $F_{4,3}$, developed at $\lambda=4$, with range of values $(2,4)$ along the real axis, $F_{4,5}$, developed at $\lambda=4$, with range of values $(4, \infty)$ along the real axis, and their inverse functions. All these functions are plotted in Figure 2, their behavior along the real axis is shown in Figure 3 .

3.1. The inverse super-exponential below 2. The inverse function of $F_{2,1}(z)=$ $v\left(s_{2,1} \mathrm{e}^{\kappa_{2} z}\right)+\lambda$ is given by

$$
F_{2,1}^{-1}(z)=\log \left(V(z-\lambda) / s_{2,1}\right) / \kappa_{2}
$$

where $V$ is the Schröder powerseries of $h[2](z):=h(z+2)-2=2\left(\sqrt{2}^{z}-1\right)$. The function $F_{2,1}^{-1}$ is shown at the top of the right-hand side of Figure 2. We calculate the first few coefficients according to formulas (3.7) and (2.15):

$$
\begin{array}{lcl}
V_{1}= & 1, & \\
V_{2}= & -v_{2}=\frac{1}{4} \frac{\ln (2)}{1-\ln (2)} & \approx 0.56472283831773236365, \\
V_{3}= & \frac{\ln (2)^{2}}{24} \frac{1+2 \ln (2)}{(1-\ln (2))^{2}(1+\ln (2))} & \approx 0.29964618138408807683 .
\end{array}
$$

More values are printed in the right column of the Table 1

The series can be truncated, keeping the term of the $N$ th power, giving the approximation

$$
\tilde{F}_{2,1, N}^{-1}(z)=\frac{1}{\kappa_{2}} \log \left(\frac{1}{s_{2,1}} \sum_{n=1}^{N} V_{n} \cdot(z-2)^{n}\right) .
$$

It is useful at small values $|z-2|$. The range of approximation can be extended with the function

$$
\tilde{F}_{2,1}^{-1}(z)=\left\{\begin{array}{cc}
\tilde{F}_{2,1, N}^{-1}(z), & |z-2| \leq \rho, \\
\tilde{F}_{2,1}^{-1}\left(\exp _{b}(z)\right)-1, & |z-2|>\rho .
\end{array}\right.
$$

In particular, for $N=20$ and $\rho=0.4$, the approximation $\tilde{F}_{2,1}^{-1}$ returns of order 14 correct decimal digits and can be used for the complex $\langle$ double $\rangle$ implementation in numerical analysis. 

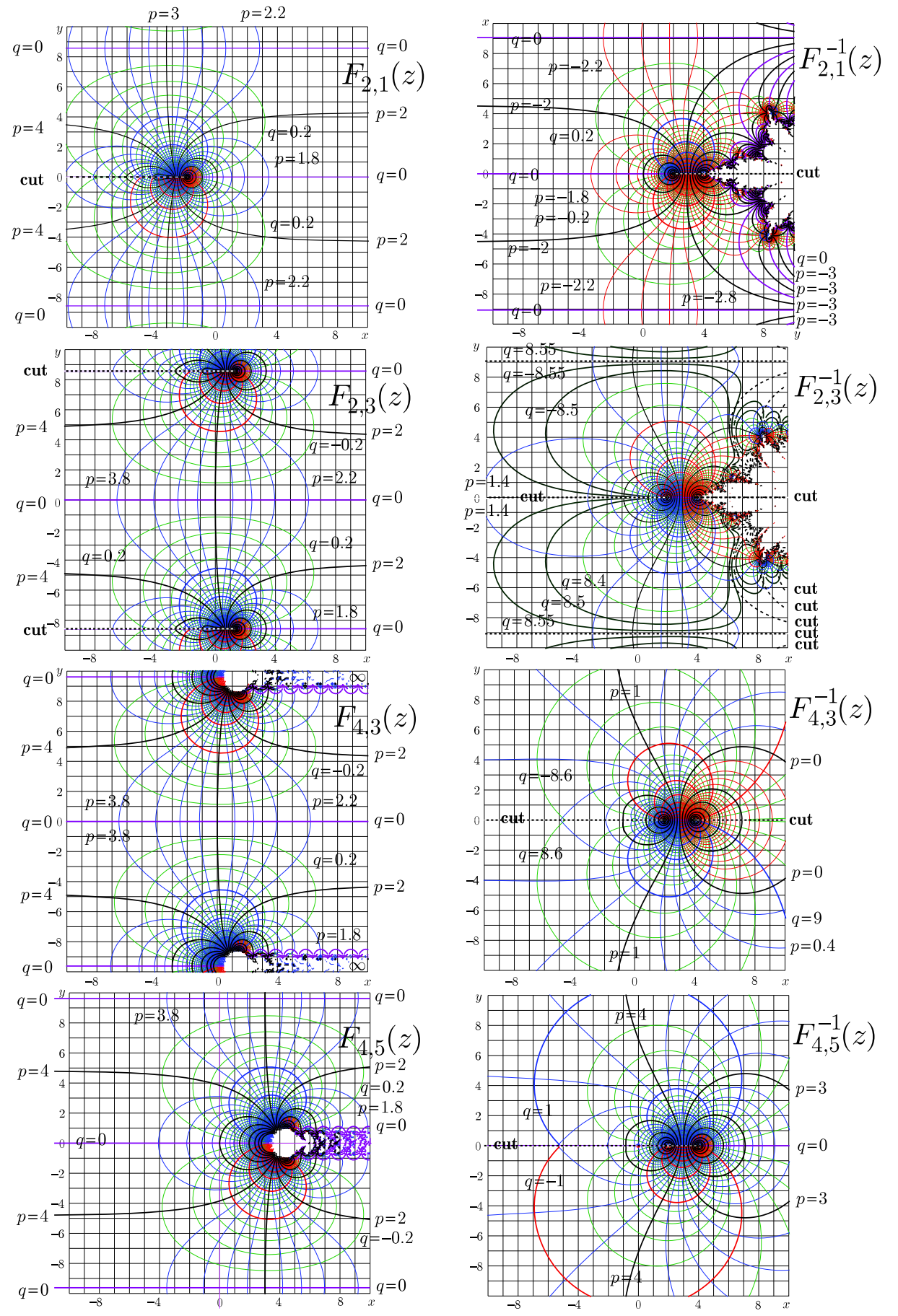

Figure 2. Functions $f=F_{2,1}(z), f=F_{2,3}(z), f=F_{4,3}(z), f=$ $F_{4,4}(z)$, left, and their inverse functions, right, in the $z=x+\mathrm{i} y$ plane, in the same notations as in Figure 1. 


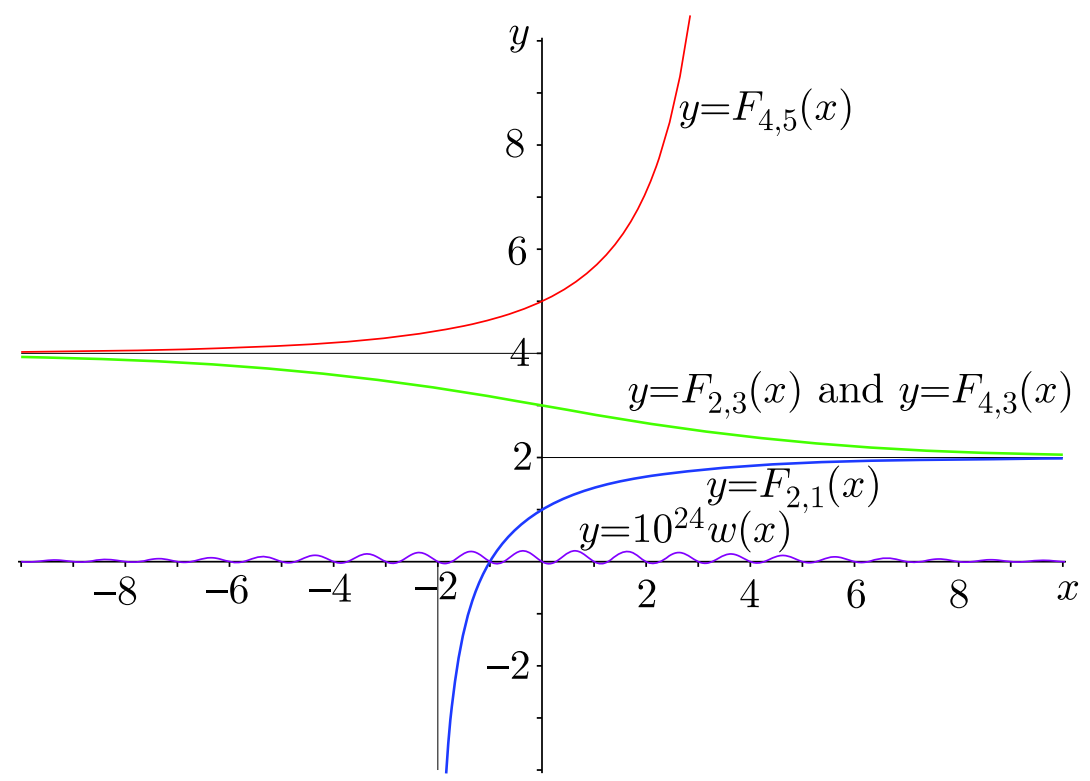

Figure 3. $F_{2,1}$ by (3.9), $F_{2,3}$ by (5.1) (which coincides with $F_{4,3}$ by (5.2) with 24 significant figures), and $F_{4,5}$ by (4.9). The asymptotes are shown with thin lines. The difference $w$ by (5.11) scaled with factor $10^{24}$ is shown with the oscillating curve in the vicinity of the abscissa axis.

The plots of functions $F_{2,1}$ and $F_{2,1}^{-1}$ look similar. This similarity indicates approximate symmetry $x \leftrightarrow-y$ of the graph $y=F_{2,1}(x)$; this function is shown again in left-hand side of Figure 7 indeed, it looks pretty symmetric. We analyze this visual effect in Section 8 .

\section{Super-exponential above FiXed POINT 4}

In this section we have

$$
\begin{aligned}
b & =\sqrt{2}, \quad h(z)=\exp _{b}(z), \quad \lambda=4, \\
K_{4} & =h^{\prime}(\lambda)=\exp _{\sqrt{2}^{\prime}}(4)=4 \ln (\sqrt{2})=2 \ln (2) \approx 1.3862943611199, \\
\kappa_{4} & =\ln \left(K_{4}\right) \approx 0.32663425997828098238
\end{aligned}
$$

With formula (2.15) we compute the powerseries $u$ of the inverse Schröder function of $h[4](z):=h(z+4)-4$ :

$$
\begin{aligned}
h[4](z) & =4\left(\exp \left(\frac{\ln (2)}{2} z\right)-1\right)=2 h[2](z), \\
h[4](z) & =\sum_{n=1}^{\infty} \frac{\ln (2)^{n}}{2^{n-2} n !} z^{n} \\
h[4]_{1} & =2 \ln (2), h[4]_{2}=\frac{\ln (2)^{2}}{2}, h[4]_{3}=\frac{\ln (2)^{3}}{12}, \ldots,
\end{aligned}
$$


TABle 2. Coefficients $u$ and $U$ in expansions (4.9) and (4.22)

\begin{tabular}{rcr}
$n$ & $u_{n}$ & \multicolumn{1}{c}{$U_{n}$} \\
1 & 1.0000000000000000 & 1.0000000000000000 \\
2 & 0.4485874311952612 & -0.4485874311952612 \\
3 & 0.1903722467978068 & 0.2120891200549197 \\
4 & 0.0778295765369683 & -0.1021843675069717 \\
5 & 0.0309358603057080 & 0.0496986830373718 \\
6 & 0.0120221257690659 & -0.0243075903261196 \\
7 & 0.0045849888965617 & 0.0119330883965109 \\
8 & 0.0017207423310577 & -0.0058736976420089 \\
9 & 0.0006368109038799 & 0.0028968672871058 \\
10 & 0.0002327696003030 & -0.0014309081060793 \\
11 & 0.0000841455118381 & 0.0007076637148566 \\
12 & 0.0000301156464937 & -0.0003503296158730 \\
13 & 0.0000106807458130 & 0.0001735756004664 \\
14 & 0.0000037565713616 & -0.0000860610119291 \\
15 & 0.0000013111367785 & 0.0000426959089013 \\
16 & 0.0000004543791625 & -0.0000211930290682 \\
17 & 0.0000001564298463 & 0.0000105244225996 \\
18 & 0.0000000535232764 & -0.0000052285174354 \\
19 & 0.0000000182077863 & 0.0000025984499916 \\
20 & 0.0000000061604765 & -0.0000012917821121
\end{tabular}

$$
\begin{aligned}
& u_{2}=\quad \frac{\ln (2) / 4}{2 \ln (2)-1} \quad \approx 0.44858743119526122890, \\
& u_{3}=\frac{(1+\ln (2)) \ln (2)^{2} / 12}{1-2 \ln (2)-4 \ln (2)^{2}+8 \ln (2)^{3}} \quad \approx 0.19037224679780675668 .
\end{aligned}
$$

More values for the coefficients $u$ are printed in Table 2 .

While in the case $\lambda=2$ the preferable initial condition is $F_{2,1}(0)=1$, which implies

$$
F_{2,1}(n)=\exp _{\sqrt{2}}^{[n]}(1)=\underbrace{\sqrt{2}}_{n \times \sqrt{2}},
$$

we see no evident preference for $\lambda=4$. So we choose $F(0)=5$ for the superexponential above 4 . From (2.22), for $\eta=u$, we get

$$
\begin{aligned}
F_{4,5}(z) & =u\left(s_{4,5} \mathrm{e}^{\kappa_{4} z}\right)+4, \\
s_{4,5} & =U(5-4)=U(1) \approx 0.69470714714324 .
\end{aligned}
$$

In analogy with $v$ and $V$, let $U$ denote the inverse function of $u$; and let $F_{4,5}$ denote the corresponding super-exponential. The choice $F_{4,5}(0)=5$ allows the interpretation of $F_{4,5}(n)$ as a result of $n$ applications to number 5 of the exponential to base $\sqrt{2}$ :

$$
F_{4,5}(n)=\exp _{\sqrt{2}}^{[n]}(5)=\underbrace{\sqrt{2} \cdot \cdots \sqrt{2}^{5}}_{n \text { repetitions of } \sqrt{2}},
$$



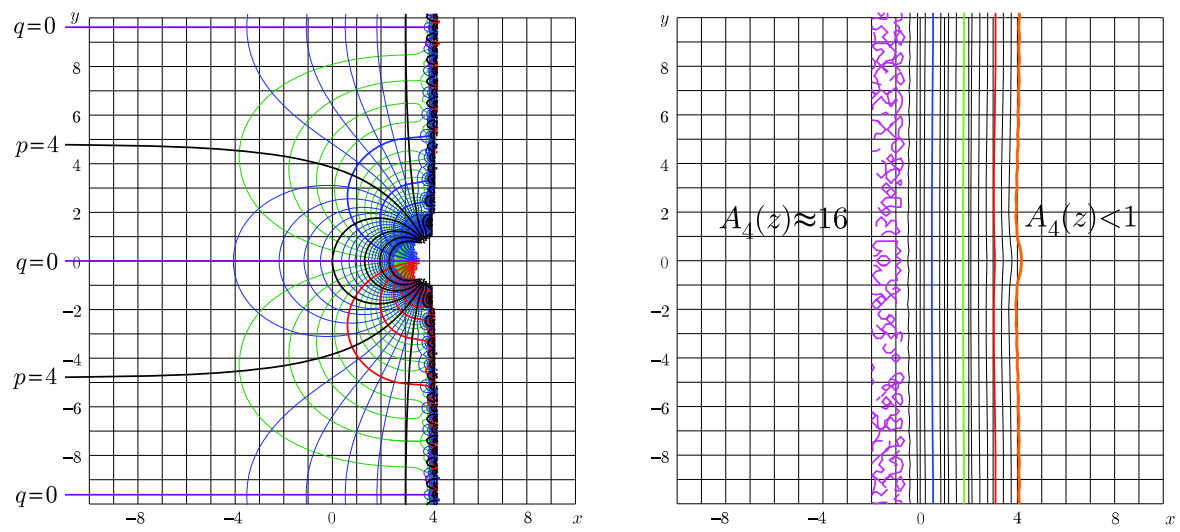

Figure 4. Left: Function $f=F_{4,5}(z)$ in the $x+\mathrm{i} y$ plane; levels $p=\Re(f)=$ const and levels $q=\Im(f)=$ const are shown with thick lines for integer values of $p$ and $q$. Right: Function $f=A_{4}(z)$ by (4.13) in the $x+\mathrm{i} y$ plane; levels $f=1,4,8,12,16$ are shown with thick lines.

at least for natural numbers $n$.

Note, that the super-exponentials below 2 , for example $F_{2,1}$, remain smaller than 2 along the real axis, actually in the interval $(-2, \infty)$, as we did not define it for $(-\infty,-2]$; but the super-exponentials above 4 , for example $F_{4,5}$, remain larger than 4 along the whole real axis. The super-exponential below 4 will be considered later together with the super-exponential above 2; along the real axis, these functions are also plotted in Figure 3 .

For the evaluation of (4.9) and (4.10), in analogy with super-exponential developed at $\lambda=2$, we truncate the powerseries. The truncation of the powerseries $u$, keeping $N+1$ terms, leads to the following approximation, let us call it $\tilde{F}_{4,5, N}$, of the super-function $F_{4,5}$ :

$$
\tilde{F}_{4,5, N}(z)=4+\sum_{n=1}^{N} u_{n} s_{4,5}^{n} \exp \left(n \kappa_{4} z\right)
$$

The function $\tilde{F}_{4,5,20}$ is plotted in the complex $z$-plane in the left-hand side of Figure 4. In the right part of the left picture, the density of the levels is so high, that the plotter cannot draw them; this part is left empty.

In order to characterize the precision of the approximation of solution $F_{4,5}$ of equation (1.1), in the right-hand side of Figure 4, the agreement

$$
A_{4}(z)=-\lg \left|\frac{\tilde{F}_{4,5,20}(z)-\exp _{b}\left(\tilde{F}_{4,5,20}(z-1)\right)}{\tilde{F}_{4,5,20}(z)+\exp _{b}\left(\tilde{F}_{4,5,20}(z-1)\right)}\right|
$$

is plotted. The plot indicates, that, at $\Re(z)<-1$, the error of evaluation is determined by the rounding errors of the complex $\langle$ double $\rangle$ arithmetic, not by the error of the approximation $\tilde{F}_{4,5,20}$. For the approximation of the solution $F_{4,5}$ in the whole complex plane, we suggest the function

$$
\tilde{F}_{4,5}(z)=\left\{\begin{array}{cl}
\tilde{F}_{4,5, N}(z), & \Re(z)<x_{N}, \\
\exp _{b}\left(\tilde{F}_{4,5}(z-1)\right), & \Re(z) \geq x_{N} .
\end{array}\right.
$$


For $N=20$ and $x_{20}=-1$ it returns of order of 14 significant figures of function $F_{4,5}$. This approximation is used to plot $F_{4,5}$ in Figures 2 and 3 . The evaluation can be performed also at smaller values of $N$; then, for good precision we need to choose $\left|x_{N}\right|>\left|x_{20}\right|$.

In the left-hand side of the complex plane, $F_{4,5}$ approaches the fixed point $\lambda=4$. Along the real axis, the function grows faster than any exponential, and it shows the fractal behavior in the vicinity of the positive part of the real axis. At moderated distances from the real axis, the function $F_{4,5}$ changes slowly, approaching the fixed point $\lambda=2$.

The function $F_{4,5}$ is periodic; the period $T_{4}$ is expressed by

$$
T_{4}=\frac{2 \pi \mathrm{i}}{\kappa_{4}} \approx 19.236149042042854712 \mathrm{i},
$$

so, at the distance of order of 19 from the real axis, the fractal structure is reproduced, although it is out of range of its plot in Figure 2. The function shows rapid growth in the positive part of the real axis and complicated behavior in its vicinity, but it is analytic in every point of the complex plane:

Proposition 11. $F_{4,5}$ is entire.

Proof. $h=\exp _{\sqrt{2}}$ is entire and $K=2 \ln (2)>1$. So Proposition 4 can be applied.

However, the growth of the entire $F_{4,5}$ in the vicinity of the real axis is so rapid, that even in the range of the plot in Figure 2, the values cannot be stored in a complex $\langle$ double $\rangle$ variable; there values are marked with the symbol $\infty$. Such a rapid growth is typical for the growing super-exponentials.

4.1. Inverse super-exponential above 4. The inverse function $F_{4,5}^{-1}$ of function $F_{4,5}$ can be defined with the relations

$$
\begin{aligned}
F_{4,5}\left(F_{4,5}^{-1}(z)\right) & =z \forall z \in \mathbb{C} \backslash\{x \in \mathbb{R}: x \leq 4\}, \\
\left|\Im\left(F_{4,5}^{-1}(z)\right)\right| & <\Im\left(T_{4}\right) / 2 \approx 9.6180745210214273558 .
\end{aligned}
$$

It is shown at the bottom of Figure 2. The range of validity of the reciprocal relation

$$
F_{4,5}^{-1}\left(F_{4,5}(z)\right)=z
$$

is narrower than $\mathbb{C} \backslash\{x \in \mathbb{R}: x \leq 4\}$; it is limited by a single period of function $F_{4,5}$ along the imaginary axis. The function $F_{4,5}^{-1}(z)$ has only two singularities at $z=2$ and $z=4$, we choose the cut line along the real axis, $z<4$. In the positive direction of the real axis, the function slowly grows, slower than a logarithm. We consider the properties of this function and its evaluation in this section.

The inverse function satisfies the Abel equation [1, 3]

$$
F_{4,5}^{-1}\left(\exp _{b}(z)\right)=F_{4,5}^{-1}(z)+1 .
$$

This function can be expressed with the Schröder powerseries $U$ of $h[4]$ :

$$
F_{4,5}^{-1}(z)=\log \left(U(z-4) / s_{4,5}\right) / \kappa_{4} .
$$


We compute first some coefficients of $U$ with formula (2.15):

$$
\begin{array}{lcl}
U_{1}= & 1, & \\
U_{2}= & -u_{2}=\frac{\ln (2) / 4}{1-2 \ln (2)} & \approx-0.4485874311952612289, \\
U_{3}= & \frac{(4 \ln (2)+1) / 24}{1-2 \ln (2)-4 \ln (2)+8 \ln (2)^{2}} & \approx 0.21208912005491969757 .
\end{array}
$$

More values are printed in the second column of Table 2. Numerically, the inverse function can be implemented with

$$
\tilde{F}_{4,5, N}^{-1}(z)=\frac{1}{\kappa_{4}} \log \left(\frac{1}{s_{4,5}} \sum_{n=1}^{N} U_{n} \cdot(z-4)^{n}\right) ;
$$

this approximation is useful for small values of $|z-4|$. The range of the approximation can be extended with the function

$$
\tilde{F}_{4,5}^{-1}(z)=\left\{\begin{array}{cc}
\tilde{F}_{4,5}^{-1}\left(\log _{b}(z)\right)+1, & |z-4|>\rho, \\
\tilde{F}_{4,5, N}^{-1}(z), & |z-4| \leq \rho .
\end{array}\right.
$$

At $N=20$ and $\rho=0.4$ such an approximation returns at least 14 significant figures and can be recommended for a complex〈double $\rangle$ implementation.

\section{Super-exponentials ABOVe 2 And Below 4}

In this section, we consider the two remaining classes of super-exponentials to base $\sqrt{2}$. These are exemplary given by

$$
\begin{aligned}
& F_{2,3}(z)=v\left(s_{2,3} \mathrm{e}^{\kappa_{2} z}\right)+2, \quad s_{2,3}=V(3-2)=V(1) \approx 2.1844747586390 \\
& F_{4,3}(z)=u\left(s_{4,3} \mathrm{e}^{\kappa_{4} z}\right)+4, \quad s_{4,3}=U(3-4)=U(-1) \approx-1.8604041940071
\end{aligned}
$$

$s$ is chosen such that $F_{2,3}(0)=F_{4,3}(0)=3$.

Every regular super-exponential $F(z)$ at fixed point $\lambda$ is a translation in the $z$-plane of the principal (see Definition 3 and Corollary 8) super-exponential. If $s$ is positive, it is a real translation, otherwise it is a complex translation. The superexponential with $s=-1$ can be obtained by a translation along the imaginary axis by half of the corresponding period $T=2 \pi \mathrm{i} / \kappa$ :

$$
-\mathrm{e}^{\kappa z}=\mathrm{e}^{\kappa z+\pi \mathrm{i}}=\mathrm{e}^{\kappa(z+\pi \mathrm{i} / \kappa)}=\mathrm{e}^{\kappa(z+T / 2)} .
$$

For systematization of our super-exponentials, let us begin with the case, when, in (2.22), the Schröder parameter $s$ is unity. We call the corresponding superfunction "principal super-exponential" or, if we need to indicate the value of $\kappa$, "the $\kappa$-principal super-exponential". Let $\mathcal{F}_{\lambda}$ denote the principal super-exponential at $\lambda$, i.e., $\mathcal{F}_{\lambda}(z)=\eta\left(\exp \left(\kappa_{\lambda} z\right)\right)+\lambda$; we use this expression for functions $\eta=u$ and $\eta=v$. Then, all our super-exponentials can be expressed as principal super-exponentials with displaced arguments:

$$
\begin{array}{ll}
F_{2,1}=\mathcal{F}_{2}\left(z+t_{2,1}+T_{2} / 2\right) & t_{2,1}=\frac{\ln \left|s_{2,1}\right|}{\kappa_{2}} \approx 1.25155147882219, \\
F_{2,3}=\mathcal{F}_{2}\left(z+t_{2,3}\right) & t_{2,3}=\frac{\ln \left(s_{2,3}\right)}{\kappa_{2}} \approx-2.13191778709503, \\
F_{4,3}=\mathcal{F}_{4}\left(z+t_{4,3}+T_{4} / 2\right) & t_{4,3}=\frac{\ln \left|s_{4,3}\right|}{\kappa_{4}} \approx 1.90057764535872, \\
F_{4,5}=\mathcal{F}_{4}\left(z+t_{4,3}\right) & t_{4,5}=\frac{\ln \left(s_{4,5}\right)}{\kappa_{4}} \approx-1.11520724513161 .
\end{array}
$$



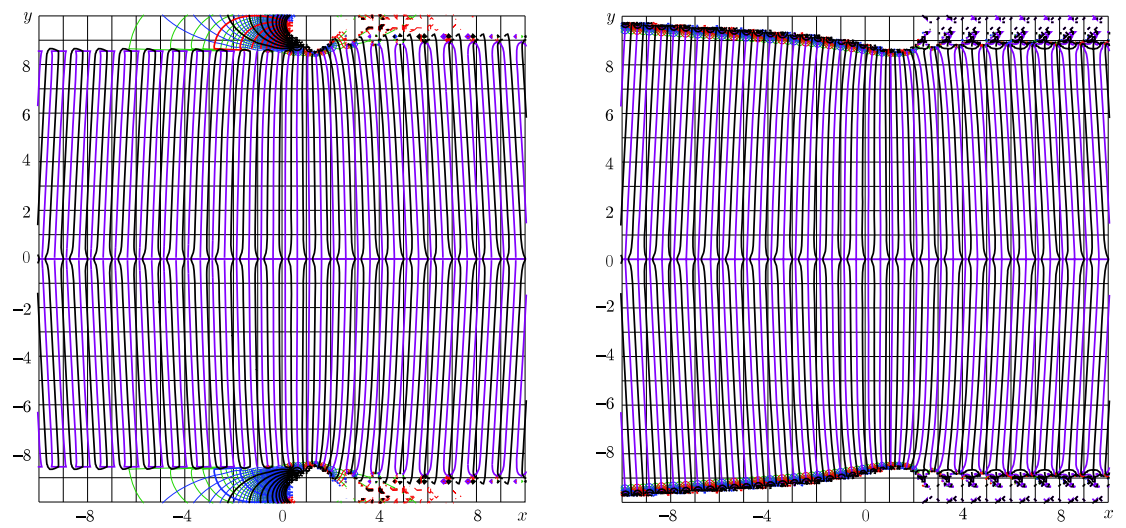

Figure 5. Functions $w$ and $\tilde{w}$ by (5.11) in the complex plane

In consequence the functions $F_{2,3}$ and $F_{4,3}$ correspond to a complex translation of the functions $F_{2,1}$ and $F_{4,5}$, respectively:

$$
\begin{aligned}
& F_{2,3}(z)=F_{2,1}\left(z+t_{2,3}-t_{2,1}-T_{2} / 2\right), \\
& F_{4,3}(z)=F_{4,5}\left(z+t_{4,3}-t_{4,5}-T_{4} / 2\right) .
\end{aligned}
$$

We place the cut lines of $F_{2,3}^{-1}$ and $F_{4,3}^{-1}$ along the half-lines $\Re(z)<2$ and $\Re(z)>4$, in order to keep them analytic in the vicinity of $z=3 . F_{2,3}$ and $F_{4,3}$ are shown in the left-hand side of Figure 2 and their inverse are plotted in the right-hand side.

The plots for $F_{2,3}$ and $F_{4,3}$ look similar. At least for $\Im(z)<T_{2} / 2$, the two $(0 \mapsto 3)$ super-exponentials are related to

$$
F_{2,3}(z)=F_{4,3}\left(z+\theta_{2,4}(z)\right), \quad \theta_{2,4}(z)=F_{4,3}^{-1}\left(F_{2,3}(z)\right)-z
$$

where $\theta_{2,4}$ is a 1-periodic function. At least in the vicinity of the real axis, it can be approximated with the sinusoidal formula

$$
\theta_{2,4}(z) \approx \tilde{\theta}_{2,4}(z)=\xi \cdot(\sin (\phi+2 \pi z)-\sin (\phi))
$$

with parameters $\xi=0.7252175546 \cdot 10^{-24}$ and $\phi=0.74633983476$. The approximation $\tilde{\theta}_{2,4}$ can be used for the complex $\langle$ double $\rangle$ implementation of $w(z)=F_{4,3}(z)-$ $F_{2,3}(z)$ for $|\Im(z)|<4$ :

$$
w(z)=F_{4,3}(z)-F_{2,3}(z) \approx \tilde{w}(z):=\tilde{\theta}_{24}(z) \cdot F_{4,3}{ }^{\prime}(z) .
$$

Function $w$ is plotted in the left-hand side of Figure [5] and function $\tilde{w}$ in the righthand side. The plots of $w$ and $\tilde{w}$ look identical at moderate values of the imaginary part of the argument. In addition, each of them is zero, while the argument is integer. However, the entire function $\tilde{w}$ reproduces neither the cut lines, nor the singularities of function $w$; and the deviation of $\tilde{w}$ from $w$ becomes visible at the distance of order of $\left|T_{2}\right| / 2 \approx 8.5$ from the real axis.

Any small periodic or quasi-periodic perturbation of a holomorphic function, real along the real axis, grows up in the direction of the imaginary axis. The quasiperiod unity determines the increment of this growth and is of the order of $2 \pi$. In the case of function $w$, this perturbation becomes of the order of unity at the distance of the order of $\left|T_{2}\right| / 2$. This allows us to estimate the smallness of the deviation $w$ in the vicinity of the real axis. At the distance of order $\left|T_{2}\right| / 2$ from 
the real axis, the quasi-periodic perturbation of the function grows up until unity (and even becomes infinite at the singularities of $F_{2,3}$ ). This gives an estimate of the order of magnitude of this deviation; for moderate values of $\Im(z)$, i.e., in the vicinity of the real axis,

$$
\begin{aligned}
|w(z)|<\sigma & :=\exp \left(-\pi\left|T_{2}\right|\right)=\exp \left(\frac{-2 \pi^{2}}{|\ln (\ln (2))|}\right) \\
& \approx \exp (-53.8) \approx 4 \cdot 10^{-24} .
\end{aligned}
$$

The perturbation is not exactly sinusoidal. As we leave the real axis, the highest harmonics grow faster; so, we may expect, that, at the real axis, the deviation is smaller than $\sigma$. The function $10^{24} w(x)$ versus real $x$ is shown at the bottom of Figure 3. it oscillates in the vicinity of the real axis and, indeed, for real $x$ the values of $|w(x)|$ are an order of magnitude smaller than $\sigma$.

In such a way, the order of magnitude of the deviation between the two $(C, 0 \mapsto 3)$ super-functions of $\exp _{b}$ is determined by their periods, which, in turn, is determined by the derivatives of the exponentials at the fixed points $\lambda=4$ and $\lambda=2$ for $b=\sqrt{2}$.

The deviation $\mathcal{D}$ by (7.3) of the iterated exponentials, made of super-function with periods $T_{4}$ and $T_{2}$ is also smaller than $\sigma$ in the $(2,4)$ interval; this deviation is considered in Section 7 . The deviation between two holomorphic functions cannot remain small everywhere (while this deviation is not a constant); therefore it can be seen comparing the maps of these functions in the complex plane. The extension to the complex plane is a universal tool for the analysis of functions that differ only a little at the real axis.

\section{Periodicity of inverse Super-exponentials}

When considering inverse holomorphic super-functions in the complex plane one is tempted to make some mistakes of the same category as the branch-related mistake $\log (a b)=\log (a)+\log (b)$ for $a, b \in \mathbb{C} \backslash\{0\}$. One merely can state that for each $a$ and $b$ we can choose a branch of the logarithm on the left side such that the previous equation is true. Or one can restrict the validity of the equation to the right half-plane.

Without going too deep into the theory of global holomorphic functions, we want to mention this source of confusion. Especially for derivations like:

$$
\begin{aligned}
F(z+1) & =h(F(z)), \\
F^{-1}(z)+1 & =F^{-1}(h(z)) .
\end{aligned}
$$

The inverse of a super-function may be an Abel-function only on some restricted domain or up to choosing the correct branch.

Moreover, for inverse real-analytic super-exponentials $G$ the following derivation is correct only up to choosing the proper branch. Let $T=2 \pi \mathrm{i} / \ln (b)$ be the period of $z \mapsto b^{z}$ :

$$
\begin{aligned}
G\left(b^{z}\right) & =G(z)+1, \\
G(z)+1=G\left(b^{z}\right) & =G\left(b^{z+T}\right)=G(z+T)+1, \\
G(z) & =G(z+T) .
\end{aligned}
$$

Proposition 12. Let $G$ be the inverse (taken along the real axis and analytically continued) of a regular super-exponential to base $b \in\left(1, \mathrm{e}^{1 / \mathrm{e}}\right)$, then $G$ is periodic 
such that

$$
G(z+T)=G(z), \quad T=\frac{2 \pi \mathrm{i}}{\ln (b)},
$$

up to choosing a suitable branch of $G$.

From the considered inverse super-exponentials — with cuts as indicated - the only properly periodic function is $F_{2,1}^{-1}$, i.e.,

$$
F_{2,1}^{-1}\left(z+T_{\text {slog }}\right)=F_{2,1}^{-1}(z), \quad T_{\text {slog }}=\frac{4 \pi \mathrm{i}}{\ln (2)} \approx 18.129440567308775239 \mathrm{i}
$$

for each $z \neq(2, \infty)+T_{\operatorname{slog}} k, k \in \mathbb{Z}$.

The period $T_{\text {slog }}$ is determined by the jump of the function $F_{2,1}$ at the cut between points -1 and -2 ; which is of the same order of magnitude as the period of the super-exponential $F_{4,5}$ by (4.15) and the period $T_{2}$ of the tetrational by (3.15). The closeness of the periods $T_{2}$ to the period $T_{\text {slog }}$ seems to be a specific property of the tetrational to base $\sqrt{2}$. In particular, such a closeness allows functions $F_{2,1}$ and $x \mapsto-F_{2,1}{ }^{-1}(-x)$ to be nearly identical along the real axis; this topic is considered in Section 8

\section{Non-Integer iteration of the EXPOnential to Base $\sqrt{2}$}

The pair $F_{\lambda, d}$ and $F_{\lambda, d}^{-1}$ can be used to define fractional iteration of the exponentiation to the base $b=\sqrt{2}$. For $\lambda=4$, we define

$$
\exp _{b, 4}^{[c]}(z)=F_{4, d}\left(c+F_{4, d}^{-1}(z)\right) \forall z \in \mathbb{C} \backslash\{x \in \mathbb{R}: x \leq 4\} .
$$

In particular, we may use $d=3$ or $d=5$ in (7.1).

Due to relation (5.8), the displacement of the argument of function $F_{\lambda, d}$ is compensated by the displacement of the value of function $F_{\lambda, d}^{-1}$. For this reason, we do not specify the value of $d$ in the left-hand side of (7.1); it does not depend on $d$.

For $c=0.5$ the $\exp _{b, 4}^{[c]}$ by (7.1) is shown in the right-hand side of Figure 6 in the same notation as in Figure 1 .

However, the shape of the iterated exponential depends on the fixed point $\lambda$ used to construct the super-functions. The pair of functions $F_{2, d}$ and $F_{2, d}^{-1}$ also allows us to construct the iterated exponential

$$
\exp _{b, 2}^{[c]}(z)=F_{2, d}\left(c+F_{2, d}^{-1}(z)\right)
$$

in a similar way as is constructed with the pair $F_{4, d}$ and $F_{4, d}^{-1}$ in equation (7.1). The range of holomorphism is a little bit more complicated than in the case $\lambda=4$ because of the complicated behavior of the $F_{2, d}^{-1}$ shown in the right-hand side of Figure 2. Similarly, due to relation (5.7), we can use any of the already described functions, either $d=1$ or $d=3$, and get the same $\exp _{b, 2}^{[c]}$. For $c=0.5$, this function is plotted in the left-hand side of Figure 6.

The functions $\exp _{\sqrt{2}, 2}^{[c]}$ and $\exp _{\sqrt{2}, 4}^{[c]}$ versus real argument are plotted in the bottom, right picture of Figure [6. In the range $2<x<4$ these curves overlap. (They were expected to overlap, because, along the real axis, the functions $F_{2,3}$ and $F_{4,3}$ overlap with 24 significant figures; see Figure 3 ). 

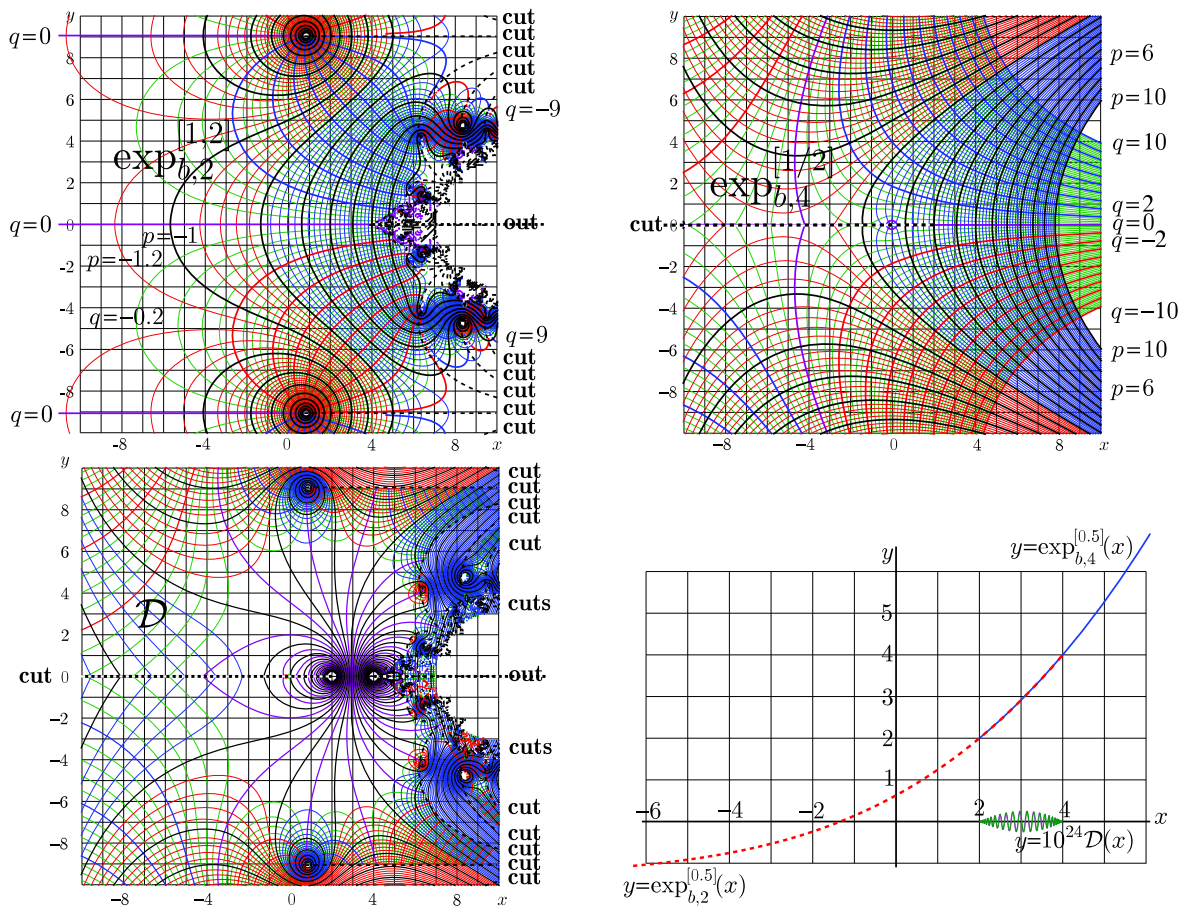

Figure 6. Comparison of functions $\exp _{b, 4}^{[c]}$ (top, left) and $\exp _{b, 2}^{[c]}$ (top, right) for $c=0.5$; difference $\mathcal{D}$ (left,bottom) in the complex plane; and both functions versus real argument (right, bottom): $y=\exp _{b, 4}^{[c]}(x)$ by [7.1, solid, and $y=\exp _{b, 2}^{[c]}(x)$ by [7.2, dashed; function $\mathcal{D}$ by (7.3), scaled with factor $10^{24}$, oscillates in the vicinity of the abscissa axis, $2<x<4$.

In spite of the similar values along the real axis, functions $\exp _{\sqrt{2}, 4}^{[0.5]}$ and $\exp _{\sqrt{2}, 2}^{[0.5]}$ are different. Function $\exp _{b, 4}^{[c]}(z)$ has a single cut-line at $z<2$. Function $\exp _{b, 2}^{[c]}(z)$ has two sets of cut-lines

$$
\Re(z)>4, \Im(z)=\Im\left(T_{\text {slog }}\right) n, n \in \mathbb{N} ;
$$

and

$$
\Re(z)>\Re\left(F_{2,1}\left(-\frac{5}{2} \pm o \mathrm{i}\right)\right) \approx 0.8386449382477, \Im(z)=\Im\left(T_{\text {slog }}\right) \frac{2 n+1}{2}, n \in \mathbb{N} ;
$$

also, this function has a fractal of singularities and additional cut-lines at $\Re(z)>4$, due to the singular behavior of function $F_{4,5}^{-1}(z)$ in that region.

Function $\exp _{b, 4}^{[c]}(z)$ is not periodic, as $F_{4,5}^{-1}$ is not periodic.

Function $\exp _{b, 2}^{[c]}(z)$ is periodic, as $F_{2,1}^{-1}$ is periodic; the period $T_{\text {slog }}$ is determined by (6.7).

In order to characterize the deviation of the two iterated exponentials, we analyze the difference

$$
\mathcal{D}(z)=\exp _{\sqrt{2}, 4}^{[0.5]}(z)-\exp _{\sqrt{2}, 2}^{[0.5]}(z)
$$


Function $\mathcal{D}$, scaled with factor $10^{24}$ is plotted in the range $2<x<4$ in the right bottom part of Figure 6 , $|\mathcal{D}(x)|<\sigma$.

The two iterated exponentials strongly deviate from each other in the vicinity of $z=\Re\left(F_{2,1}(-2.5+o \mathrm{i})\right)+\mathrm{T}_{\text {slog }} / 2$, where the function $\exp _{b, 2}^{[c]}(z)$ has a singularity. (Symbol oi indicates that the function should be evaluated just above the cut-line.)

For $|z-3|<1$, the function $\mathcal{D}(z)$ can be roughly approximated with the 6parametric fit

$$
\begin{aligned}
& \tilde{\mathcal{D}}(z)=2.48 \cdot 10^{-25}(z-2)(4-z)\left(1+0.120(z-3)+0.006(z-3)^{2}\right) \\
& \times \sin \left(0.747-0.068(z-3)+0.007(z-3)^{2}+T_{4} \ln (4-z)+T_{2} \ln (z-2)\right) .
\end{aligned}
$$

The constants $T_{2}$ and $T_{4}$ are determined by (3.15) and (4.15). In Figure 7 both functions $10^{24} \mathcal{D}$ and $10^{24} \tilde{\mathcal{D}}$ overlap well and the error of the approximation $\tilde{\mathcal{D}}$ is not seen even at the zooming-in. For detailed numerical analysis of the deviation between the two $(0 \mapsto 3)$ super-exponentials in the vicinity of the real axis, even a more precise fit of function $\mathcal{D}$ can be elaborated on the base of approximation (5.11) of the difference $w$ between the two super-exponentials, treating the constant $\sigma$ by (5.13) as a small parameter.

As in the case of super-exponentials $F_{2,3}$ and $F_{4,3}$ and as in the case of the iterated exponentials $\exp _{b, 2}^{[c]}$ and $\exp _{b, 4}^{[c]}$, the precise evaluation is required to see that the two functions are not the same. Dan Asimov privately communicated that he, Dean Hickerson and Richard Schroeppel recognized this deviation of the half-iterate around 1991 by computing the values to 25 digits of accuracy. The only accessible reference is his posting [ $[\underline{8}$ on sci.math in the form of an April fool's joke.

However, the deviation becomes more visible outside the real axis, functions are clearly distinguishable without any zooming-in. In the following section we consider an additional (and last) example when leaving the real axis significantly simplifies the consideration.

\section{About the apparent $(x,-y)$ Symmetry of the graph of the SUPER-EXPONENTIAL BELOW 2}

The $(x,-y)$ symmetry of graph of $y=f(x)$, apparently shown in Figure 7 for $f=F_{2,1}$, can be described by the formula

$$
f^{-1}(-x)=-f(x)
$$

or, by applying function $f$ to equation (8.1),

$$
f(-f(x))=-x .
$$

Generally, for some function $f$, there is nothing wrong in relations (8.1), (8.2). For example, each of the functions

$$
f(x)=1+x, \quad f(x)=\sqrt{25-(3-x)^{2}}-3, \quad f(x)=\frac{2 x+3}{x+2}
$$

satisfies (8.1), (8.2); and the graphic of the last of them even has the same vertical and horizontal asymptote $x=-2$ and $y=2$ (although does not approach them exponentially). In this section we show, that function $f$, satisfying (8.2), (8.1), cannot be $F_{2,1}$. 

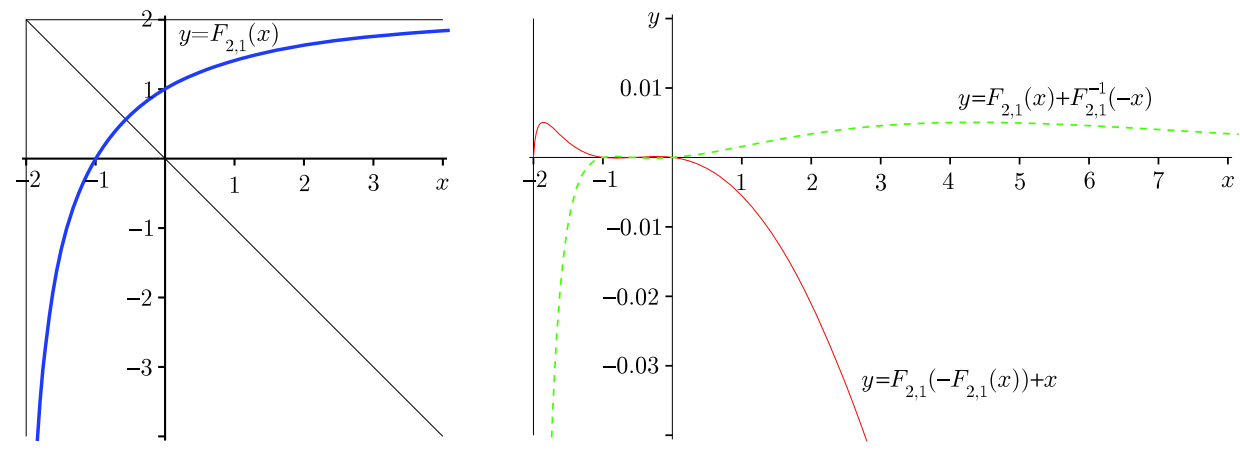

Figure 7. Function $y=F_{2,1}(x)$ of real argument left; functions $y=F_{2,1}\left(-F_{2,1}(x)\right)+x$ and $\left.y=F_{2,1}(x)+F_{2,1}^{-1}(-x)\right)$, right.

Below, we present two independent proofs. The first proof shows, that such a symmetry does not hold for a function with asymptotic behavior of function $F_{2,1}$. The second proof shows, more generally, that such a symmetry cannot hold for any super-exponential $f$ to base $\sqrt{2}$ that is monotonous on the $(-2, \infty)$ interval. Both propositions below and their proofs operate exclusively with real quantities.

Proposition 13. $y=F_{2,1}(x)$ is not $(x,-y)$ symmetric.

Proof. Here, we do not put indices on the function $f$, assuming that $f=F_{2,1}$. We show that for such a function, equation (8.2) does not hold. The deduction is based on the asymptotic properties of the function. Consider the behavior of function $f(x)$ in the vicinity of the singularity at $x=-2$ :

$$
f(-2+x)=\log _{b}(f(-1+x))=\log _{b}\left(f^{\prime}(-1) x+\mathcal{O}\left(x^{2}\right)\right) .
$$

In the right-hand side of (8.3), the expansion of $f$ begins with a linear term, because $f(-1)=0$. Using notation

$$
\Phi:=f^{\prime}(-1)=\frac{F_{2,1}{ }^{\prime}(0)}{\ln (\sqrt{2})} \approx 1.69973893,
$$

equation (8.3) can be rewritten as

$$
f(-2+x)=\log _{b}\left(\Phi x+\mathcal{O}\left(x^{2}\right)\right)=\log _{b}((\Phi x) \cdot(1+\mathcal{O}(x))) .
$$

For positive $u, v, \ln (u v)=\ln (u)+\ln (v)$; so, at least for positive $x$, we have

$$
\begin{aligned}
f(-2+x) & =\log _{b}(\Phi)+\log _{b}(x)+\log _{b}(1+\mathcal{O}(x)) \\
& =\log _{b}(x)+\log _{b}(\Phi)+\mathcal{O}(x) .
\end{aligned}
$$

Now remember the asymptotic behavior of $F_{2,1}(z)$ at large values of $\Re(z)$; from (3.9) and (3.3), we can represent $f(x)$ as follows:

$$
f(x)=2-\omega-\mathcal{O}\left(\omega^{2}\right),
$$

where $\omega=-s_{2,1} \mathrm{e}^{\kappa_{2} x}>0$. Then, consider the expansion at large positive $x$ of the expression

$$
f(-f(x))=f\left(-2+\omega+\mathcal{O}\left(\omega^{2}\right)\right) .
$$


Using the expansion (8.6) at $x=\omega+\mathcal{O}\left(\omega^{2}\right)$, we get

$$
\begin{aligned}
f(-f(x)) & =\log _{b}(\omega \cdot(1+\mathcal{O}(\omega)))+\log _{b}(\Phi)+\mathcal{O}(\omega) \\
& =\log _{b}(\omega)+\mathcal{O}\left(\omega^{0}\right) .
\end{aligned}
$$

Substituting $\omega$ to $\exp \left(\left(x+\ln \left|s_{2,1}\right|\right) \kappa_{2}\right)$, for $x \rightarrow \infty$ we get

$$
f(-f(x))=\frac{\kappa_{2} x}{\ln (\sqrt{2})}+\mathcal{O}\left(x^{0}\right)=\frac{2 \ln (\ln (2))}{\ln (2)} x+\mathcal{O}\left(x^{0}\right) ;
$$

equation (3.2) is used to get the last equality. The coefficient

$$
\frac{2 \ln (\ln (2))}{\ln (2)} \approx-1.057532745889795
$$

is close to minus unity, but not exactly minus unity. At large positive $x$, the expression $-f(-f(x))$ deviates from $x$ asymptotically linearly, with tangent of order of $6 \%$. This deviation is seen at the plot of $y=f(-f(x))+x$ in the righthand side of Figure 7

The symmetry can be negated also without reference to the asymptotic analysis, just from the observation that $f$ is a monotonous (increasing or decreasing) function. The deduction based on the sequential application of hypothesis (8.2) is presented below.

Proposition 14. There is no monotonous function $f$ on $(-2, \infty)$ that satisfies

$$
\begin{aligned}
f(0) & =1, \\
f(x+1) & =\sqrt{2}^{f(x)}, \\
f(-f(x)) & =-x .
\end{aligned}
$$

Proof. Below we show that there exist 3 points $x_{1}, x_{2}, x_{3}$ such that $x_{1}<x_{2}<x_{3}$, but $f\left(x_{1}\right)<f\left(x_{2}\right)>f\left(x_{3}\right)$. No one monotonous function is allowed to have such a property.

Let us show that there are these 3 points $x_{1}<x_{2}<x_{3}$; set $b=\sqrt{2}$. We start with the more difficult part of finding $x_{2}<x_{3}$ with $f\left(x_{2}\right)>f\left(x_{3}\right)$. Applying (8.13), we get

$$
\begin{aligned}
& f(0)=1, \\
& f(1)=b, \\
& f(2)=b^{b}=: c .
\end{aligned}
$$

It follows further that

$$
\begin{aligned}
f(4) & =b^{b^{c}}, \\
f\left(-b^{b^{c}}\right) & =-4, \\
f\left(-b^{b^{c}}+1\right) & =b^{-4},
\end{aligned}
$$

and we set

$$
x_{2}:=-b^{-4} \quad f\left(x_{2}\right)=b^{b^{c}}-1 .
$$


On the other hand, we conclude

$$
\begin{aligned}
f(-c) & =-2, \\
f(-c+1) & =b^{-2}, \\
f\left(-b^{-2}\right) & =c-1, \\
f\left(-b^{-2}+1\right) & =b^{c-1}, \\
f\left(-b^{c-1}\right) & =b^{-2}-1,
\end{aligned}
$$

and set

$$
x_{3}=-b^{c-1}+1 \quad f\left(x_{3}\right)=b^{b^{-2}-1} .
$$

Now we compare $x_{2}$ and $x_{3}$ :

$$
x_{2}=-b^{-4}=-0.25<-0.24510159 \approx-b^{c-1}+1=x_{3}
$$

and their values

$$
f\left(x_{2}\right)=b^{b^{c}}-1 \approx 0.84091087>0.84089642 \approx b^{b^{-2}-1}=f\left(x_{3}\right) .
$$

$x_{1}$ can then easily be found; say $x_{1}=-1<x_{2}$, then $f(-1)=0<f\left(x_{2}\right)$.

There is no need to depart from the real axis in order to see that the $(x,-y)$ symmetry of the graphic in the left-hand side of the Figure 7 is only an approximation, although in the range $(-1,0)$, this "symmetry" holds with 4 decimal digits. Having the complex $\langle$ double implementation of function $f$, the left-hand side of the expression (8.2) can be precisely evaluated; the residual is plotted in the right-hand side of Figure 7, confirming the approximate character of the $(x,-y)$ "symmetry".

We included both propositions above, because they are beautiful and could be good exercises for the first-grad students who do not yet deal with functions of complex variable. However, the approximate character of the $(x,-y)$ "symmetry" follows directly from the periodicity of functions $F_{2,1}$ and $F_{2,1}^{-1}$. Both functions are periodic, but their periods $T_{2}$ by (3.15) and $T_{\text {slog }}$ by (6.7) are not equivalent. If two functions, holomorphic at some domain $C$, coincide at some interval, for example, $(-1,0)$, then they coincide in the whole domain, and cannot have such different periods, assuming that they are not constant.

We have considered three pairs of "similar" functions: $\left(F_{2,3}, F_{4,3}\right)$ by equations (5.1), (5.2), shown in Figure 3 ( $\left.\exp _{\sqrt{2}, 2}^{[0.5]}, \exp _{\sqrt{2}, 4}^{[0.5]}\right)$ by equations (7.2), (17.1), shown in Figure 6, and, in conclusion, $\left(F_{2,1}, x \mapsto-F_{2,1}^{-1}(-x)\right)$ by equations (3.9), (3.16), plotted in the left-hand side of Figure 7 Here, the "similarity" means, that at the real-real plot, visually we cannot distinguish the function from its partner. In the last case (considered in this section), the difference between these functions is plotted with a dashed curve in the right-hand side of Figure 7 the float precision of evaluation is sufficient to reveal the deviation. In the first two cases, of order of 30 decimal digits should be evaluated in order to reveal the deviation along the real axis. In all examples considered, a high precision of evaluation is not required to see the deviation in the complex plane.

The different periodicity (in our case, periods $T_{2}, T_{\text {slog }}, T_{4}$ and $\infty$ ) allows us to reject the hypothesis of the coincidence of the two functions without making any numerical evaluation, without the asymptotic analysis of Proposition 13. and without the algebraic deduction of Proposition 14. 


\section{Summary AND PROSPECTS}

I. We have constructed four real super-functions $F_{\lambda, d}$ of the base-function $\exp _{\sqrt{2}}$ by (5.3)-(5.6). All these functions are periodic, their periods $T_{\lambda}$ are pure imaginary and are determined by the fixed points $\lambda=2$ and $\lambda=4 . \quad F_{2,1}$ by equation (5.3) is a tetrational. The functions are bijective on the real axis with the following (co)domains (see Figure 3):

$$
\begin{array}{ll}
F_{2,1}:(-2, \infty) \rightarrow(-\infty, 2) & \text { strictly increasing, } \\
F_{2,3}:(-\infty, \infty) \rightarrow(2,4) & \text { strictly decreasing, } \\
F_{4,3}:(-\infty, \infty) \rightarrow(2,4) & \text { strictly decreasing, } \\
F_{4,5}:(-\infty, \infty) \rightarrow(4, \infty) & \text { strictly increasing. }
\end{array}
$$

II. The complex translations of the argument relates function $F_{2,1}$ to function $F_{2,3}$; both functions are holomorphic at least in the right-hand side of the complex plane. The complex translations of the argument relates function $F_{4,3}$ to function $F_{4,5}$; both functions are entire. We describe the complex double〉 implementation for each of these functions and their inverses (see Figure 2), and that for the deviation of $F_{4,5}$ from $F_{4,3}$, see Figure 5. In the vicinity of the real axis, this deviation is of the order of $10^{-24}$. Function $F_{2,1}$ and its inverse function $F_{2,1}^{-1}$ have similar periods $T_{2}$ by (3.15) and $T_{\text {slog }}$ by (6.7); in the range $-1 \leq x \leq 0$, the deviation of $-F_{2,1}^{-1}(-x)$ from $F_{2,1}(x)$ is of order of $10^{-4}$; see Figure 7 .

III. With functions $F_{\lambda, d}$, the two different iterated exponentials $\exp _{b, 2}^{[c]}$ and $\exp _{b, 4}^{[c]}$ are constructed; they coincide at integer values of $c$, but for non-integer $c$, they have different asymptotic properties and different ranges of holomorphism. For $c=0.5$ we plot both functions in Figure 6 in the range $(2,4)$ along the real axis, the deviation between these functions is of order of $10^{-24}$, and each of these two functions can be arguably considered as a "true" $\sqrt{\exp _{\sqrt{2}}}$.

IV. The construction of the base-function $h$ for a given super-function $F$ is easier than the construction of a super-function for a given base-function. In principle the base-function is given by $h(z)=F\left(1+F^{-1}(z)\right)$. Such an "inverse" method could be used to build-up some "table of super-functions" as an analogy of a table of integrals. To the best of our knowledge, no such table for super-functions has been published. The compilation of such a table could be a matter for future research.

V. The regular iteration of the base-function at a fixed point, can be used for construction of various real super-functions for real base-functions with real fixed points. For example, there should exist at least two different holomorphic halfiterates of the factorial (i.e., $\sqrt{!}$ ); this function has been used as the logo of the Physics Department of the Moscow State University since the last century, without implementing an algorithm for the efficient evaluation. Only recently an algorithm for evaluation of such a function has been suggested [13].

\section{ACKNOWLEDGMENT}

The authors are grateful to the participants of the Tetration Forum [17] for the important questions and discussions. 


\section{Appendix A. Notations}

symbol

$A_{2}$

$A_{4}$

a

$b=\sqrt{2} \approx 1.414213562373$

$\mathbb{C}$

$C \subseteq \mathbb{C}$

$c$

$\chi=\eta^{-1}$

$\mathcal{X}(z)=s \chi(x)$

$D \subseteq \mathbb{C}$

$\mathcal{D}(\bar{z})=\exp _{b, 4}^{0.5}-\exp _{b, 2}^{0.5}$

$\tilde{\mathcal{D}}(z)$

$d$

$E(z)=F(z+\theta(z))$

$\mathrm{e}=\exp (1)=\sum_{n=0}^{\infty} 1 / n ! \approx 2.71$

$\exp (z)=\mathrm{e}^{z}$

$\exp _{b}(z)=b^{z}=\exp (\ln (b) z)$

$\exp _{b, \lambda}^{c}(z)=F_{\lambda, d}\left(c+F_{\lambda, d}^{-1}(z)\right)$

$\mathcal{E}(z)=\mathrm{e}^{(z+t) \kappa}$

$\eta=\chi^{-1}$

$\eta_{n}$

$F$

$F_{\lambda, d}$

$\mathcal{F}_{\lambda}$

$\Phi=F_{2,1}{ }^{\prime}(-1)=\frac{2}{\ln (2)} F_{2,1}{ }^{\prime}(0) \approx 1.69973893$

$(C, 0 \mapsto d)$ super-function of $\exp _{b}$ developed at $\lambda$; for ex.

general Taylor coefficient of the inverse Schröder super-function of base-function $h$, see (1.1) (2.22)

$G(\mathcal{E}(z))=F(z)-\lambda$

$g_{n}, n \in \mathbb{N}$

displaced super-function of modified argument

$H_{k, x}=\{z / k \in \mathbb{C}: \Re(z) \leq x\}$

$h$

$h_{n}$

$I$

$i=\mathrm{i}=\sqrt{-1}$

$j \in \mathbb{R}, j>0$

$K=K_{\lambda}=h_{1}=h^{\prime}(\lambda)$

$K_{2}=h^{\prime}(2)=\ln (2)$

$K_{4}=h^{\prime}(4)=2 \ln (2)$

$\kappa_{\lambda}=\ln \left(K_{\lambda}\right)$

$\kappa_{2}=\ln (\ln (2)) \approx-0.36651292058166432701$

$\kappa_{4}=\ln (2 \ln (2)) \approx 0.32663425997828098238$ $\lambda$

$\ln$

$m, n$

$\mathbb{N}$

$\mathbb{R}$

description ref.

(3.12)

(4.13)

(1.2)

(1.4)

\section{(1.1)}

(7.2)

(2.13)

(2.3)

(17.3)

(7.4)

(1.2)

(1.3)

(1.4)

(17.2)

(2.4)

(2.21)

(1.4)

(5.3)

(8.4)

(2.2)

(2.2)

Prop. 7

(1.1)

(2.1)

(2.24) used in the example $h(z)=z^{j}, F(z)=\exp \left(j^{z}\right)$

derivative of base-function $h$ at $\lambda$ derivative of $h$ at the fixed point $\lambda=2$ derivative of $h$ at the fixed point $\lambda=4$ wave-number wave-number for $\lambda=2$ wave-number for $\lambda=4$

fixed point, solution of $h(\lambda)=\lambda$ logarithm, inverse of exponentiation; $\ln =\log _{\mathrm{e}} ; \exp (\ln (z))=z$ used as integer variables set of natural numbers set of real numbers 
symbol

description $\mathcal{O}$ grows up not faster (or decays not slower), than its argument
$o$
infinitesimal parameter, $F_{2,1}(-2.5+o \mathrm{i})$ refers to upper manifold

○

$p, q$

$\pi=4 \arctan (1) \approx 3.14$ infix operation of composition of two functions dependent variables, $\Re$ and $\Im$ parts of a function $-\mathrm{i} / 2$ of period of $\exp$ various radii

real function: $\quad$ any $f$ such that $f\left(z^{*}\right)=f(z)^{*}$ for all $z$ where $f$ is defined $s=K^{t} \quad$ constant factor in the argument of the inverse Schröder

$s_{2,1}=V(-1) \approx-0.63209866105083$

$s_{2,3}=V(1) \approx 2.1844747586390$

$s_{4,3}=U(-1) \approx-1.8604041940071$

$s_{4,5}=U(1) \approx 0.69470714714324$

$\sigma=\exp \left(-2 \pi^{2} / T_{2}\right) \approx 4 \cdot 10^{-24}$

$T_{2}=2 \pi \mathrm{i} / \kappa_{2} \approx-17.143148179354847104 i$

$T_{4}=2 \pi \mathrm{i} / \kappa_{4} \approx 19.236149042042854712 i$

$T_{\text {slog }}=\frac{4 \pi \mathrm{i}}{\ln (2)} \approx 18.129440567308775239 i$

$t$

$t_{2,1}=\ln \left(s_{2,1}\right) / \kappa_{2} \approx 1.251551478822188$

$t_{2,3}=\ln \left(s_{2,3}\right) / \kappa_{2} \approx 0.880366308272851$

$t_{4,3}=\ln \left(s_{4,3}\right) / \kappa_{4} \approx 1.90057764535874$

$t_{4,5}=\ln \left(s_{4,5}\right) / \kappa_{4} \approx 1.11520724513161$

$\theta$

$\theta_{2,4}(z)=F_{4,3}^{-1}\left(F_{2,3}(z)\right)-z \approx \tilde{\theta}_{2,4}(z)$

$\tilde{\theta}_{2,4}(z)=\xi \cdot(\sin (\phi+2 \pi z)-\sin (\phi))$

$\xi=0.7252175546 \cdot 10^{-24} \approx 0.2 \sigma$

$\phi=0.74633983476$

$u$

$u_{n}, U_{n}$

$v$

$v_{n}, V_{n}$

$w(z)=F_{4,3}(z)-F_{2,3}(z)$

$\omega=\mathcal{E}(z)$

$x, y$

$z$

$\mathbb{Z}$ displacement of argument of super-function

modification of argument of super-function

Schröder parameter

Schröder parameter

Schröder parameter

Schröder parameter upper bound for $|w(x)|$ at $x \in \mathbb{R}$ period of $F_{2,1}$ and $F_{2,3}$ period of $F_{4,5}$ and $F_{4,3}$ period of $F_{2,1}^{-1}, F_{2,3}^{-1}, \exp _{b, 2}^{c}$ displacement for $F_{2,1}(0)=1$ displacement for $F_{2,3}(0)=3$ displacement for $F_{4,3}(0)=3$ displacement for $F_{4,3}(0)=5$ .

approximation of $\theta_{24}(z)$ parameter in approximation $\tilde{\theta}_{24}$ parameter in approximation $\tilde{\theta}_{24}$ inverse Schröder function for $\lambda=4$ Taylor coefficients of $G$ and $G^{-1}$ for $\lambda=4$ inverse Schröder function for $\lambda=2$ Taylor coefficients of $G$ and $G^{-1}$ for $\lambda=2$ deviation between two super-exponentials small parameter used to expand function $G$ used as independent real variables complex variable; sometimes, $z=x+\mathrm{i} y$ set of integer numbers

ref.

8.9

(2.17)

Fig.1

(3.15

(2.22)

(3.10)

(5.1)

(5.2)

(4.10)

(5.13)

(3.15

(3.15

(6.7)

(2.4)

(5.3)

(5.4)

(5.5)

(5.6)

(1.3)

(5.10)

(5.10)

5.10

(5.10)

(4.9)

Table 2

(3.9)

Table 1

(5.11)

(2.7)

Fig 1

Fig 1

\section{REFERENCES}

1. N. H. Abel. Correlative of the functional equation. Crelle's Journal, 2 (1827) 389.

2. M. Abramovich, I. Stegun. Table of special functions. National Bureau of Standards, New York, 1970.

3. G. Belitskii, Yu. Lubish. The real-analytic solutions of the Abel functional equations. Studia Mathematica, 134 (1999), 135-141. MR1688221(2000f:39022)

4. G. Belitskii and V. Nicolaevsky. Linear functional-differential equations on the line. Nonlinear Analysis, 30, Iss. 5, (1997), 2585-2593. MR1602884 (99c:34156)

5. G. Belitskii, V. Tkachenko. One-dimensional Functional Equations. Operator Theory: Advances and Applications, 144, Birkhäuser, 2003. MR1994638(2004e:39027) 
6. N. Bromer. Superexponentiation. Mathematics Magazine, 60, no. 3 (1987), 169-174. MR 1572659

7. C. C. Cowen. Iteration and the solution of functional equations for functions analytic in the unit disk. Transactions of the American Mathematical Society, 265 (1981), 69-95. MR607108 (82i:30036)

8. Dean Hickerson, Richard Schroeppel, Daniel Asimov. Are these two numbers equal? http://groups.google.com/group/sci.math/browse_thread/thread/1f2a6339171c26ab/ 2b92e8cb9b2b421d, 1991.

9. H. Kneser. Reelle analytische Lösungen der Gleichung $\varphi(\varphi(x))=\mathrm{e}^{x}$ und verwandter Funktionalgleichungen. Journal für die reine und angewandte Mathematik, 187 (1950), 56-67. MR0035385 (11:726e)

10. R.A. Knoebel. Exponentials reiterated. Amer. Math. Monthly 88 (1981), 235-252. MR610484 $(82 \mathrm{e}: 26004)$

11. D. Kouznetsov. Solution of the equation $F(z+1)=\exp (F(z))$ in the complex z-plane. Mathematics of computation, 78 (2009), 1647-1670. MR2501068

12. D. Kouznetsov. Portrait of the analytic extension of the 4 th Ackermann function in the complex plane. Preprint ILS UEC, 2008. http://www.ils.uec.ac.jp/〜dima/PAPERS/2008ackermann. pdf; http://en.citizendium.org/wiki/Image: Analytic4thAckermannFunction00.jpg

13. D. Kouznetsov, H. Trappmann. Superfunctions and sqrt of factorial. Preprint ILS UEC, 2009. http://www.ils.uec.ac.jp/ dima/PAPERS/2009supefae.pdf; Moscow University Physics Bulletin, 2010, in press.

14. M. Kuczma, B. Choczewski, R. Ger. Iterative Functional Equations. Encyclopedia of Mathematics and its applications, 1990. MR1067720 (92f:39002)

15. G. Szekeres. Regular iteration of real and complex functions. Acta Mathematica, 100 Iss. 3-4 (1958) 203-258. MR0107016(21:5744)

16. P. Walker. Infinitely differentiable generalized logarithmic and exponential functions. Mathematics of Computation, 57 (1991), 723-733. MR.1094963 (92d:33049)

17. http://math.eretrandre.org/tetrationforum/index.php

18. http://en.citizendium.org/wiki/Superfunction

19. http://en.citizendium.org/wiki/Tetration

Institute for Laser Science, University of Electro-Communications 1-5-1 ChofuGAOKA, Chofushi, TOKYo, 182-8585, JAPAN

E-mail address: dima@ils.uec.ac.jp

Henryk Trappmann, Kameruner Str. 9, 13351 Berlin, Germany

E-mail address: henryk@pool.math.tu-berlin.de 